\title{
NOETHER RESOLUTIONS IN DIMENSION 2
}

\author{
ISABEL BERMEJO, EVA GARCÍA-LLORENTE, IGNACIO GARCÍA-MARCO, AND MARCEL MORALES
}

\begin{abstract}
Let $R:=K\left[x_{1}, \ldots, x_{n}\right]$ be a polynomial ring over an infinite field $K$, and let $I \subset R$ be a homogeneous ideal with respect to a weight vector $\omega=\left(\omega_{1}, \ldots, \omega_{n}\right) \in\left(\mathbb{Z}^{+}\right)^{n}$ such that $\operatorname{dim}(R / I)=d$. In this paper we study the minimal graded free resolution of $R / I$ as $A$-module, that we call the Noether resolution of $R / I$, whenever $A:=K\left[x_{n-d+1}, \ldots, x_{n}\right]$ is a Noether normalization of $R / I$. When $d=2$ and $I$ is saturated, we give an algorithm for obtaining this resolution that involves the computation of a minimal Gröbner basis of $I$ with respect to the weighted degree reverse lexicographic order. In the particular case when $R / I$ is a 2-dimensional semigroup ring, we also describe the multigraded version of this resolution in terms of the underlying semigroup. Whenever we have the Noether resolution of $R / I$ or its multigraded version, we obtain formulas for the corresponding Hilbert series of $R / I$, and when $I$ is homogeneous, we obtain a formula for the Castelnuovo-Mumford regularity of $R / I$. Moreover, in the more general setting that $R / I$ is a simplicial semigroup ring of any dimension, we provide its Macaulayfication.

As an application of the results for 2-dimensional semigroup rings, we provide a new upper bound for the Castelnuovo-Mumford regularity of the coordinate ring of a projective monomial curve. Finally, we describe the multigraded Noether resolution and the Macaulayfication of either the coordinate ring of a projective monomial curve $\mathcal{C} \subseteq \mathbb{P}_{K}^{n}$ associated to an arithmetic sequence or the coordinate ring of any canonical projection $\pi_{r}(\mathcal{C})$ of $\mathcal{C}$ to $\mathbb{P}_{K}^{n-1}$.
\end{abstract}

Keywords: Graded algebra, Noether normalization, semigroup ring, minimal graded free resolution, CohenMacaulay ring, Castelnuovo-Mumford regularity.

\section{INTRODUCTION}

Let $R:=K\left[x_{1}, \ldots, x_{n}\right]$ be a polynomial ring over an infinite field $K$, and let $I \subset R$ be a weighted homogeneous ideal with respect to the vector $\omega=\left(\omega_{1}, \ldots, \omega_{n}\right) \in\left(\mathbb{Z}^{+}\right)^{n}$, i.e., $I$ is homogeneous for the grading $\operatorname{deg}_{\omega}\left(x_{i}\right)=\omega_{i}$. We denote by $d$ the Krull dimension of $R / I$ and we assume that $d \geq 1$. Suppose $A:=K\left[x_{n-d+1}, \ldots, x_{n}\right]$ is a Noether normalization of $R / I$, i.e., $A \hookrightarrow R / I$ is an integral ring extension. Under this assumption $R / I$ is a finitely generated $A$-module, so to study the minimal graded free resolution of $R / I$ as $A$-module is an interesting problem. Set

$$
\mathcal{F}: 0 \longrightarrow \oplus_{v \in \mathcal{B}_{p}} A\left(-s_{p, v}\right) \stackrel{\psi_{p}}{\longrightarrow} \cdots \stackrel{\psi_{1}}{\longrightarrow} \oplus_{v \in \mathcal{B}_{0}} A\left(-s_{0, v}\right) \stackrel{\psi_{0}}{\longrightarrow} R / I \longrightarrow 0
$$

this resolution, where for all $i \in\{0, \ldots, p\} \mathcal{B}_{i}$ denotes some finite set, and $s_{i, v}$ are nonnegative integers. This work concerns the study of this resolution of $R / I$, which will be called the Noether resolution of $R / I$. More precisely, we aim at determining the sets $\mathcal{B}_{i}$, the shifts $s_{i, v}$ and the morphisms $\psi_{i}$.

One of the characteristics of Noether resolutions is that they have shorter length than the minimal graded free resolution of $R / I$ as $R$-module. Indeed, the projective dimension of $R / I$ as $A$-module is $p=d-\operatorname{depth}(R / I)$, meanwhile its projective dimension of $R / I$ as $R$-module is $n$ - $\operatorname{depth}(R / I)$. Studying Noether resolutions is interesting since they contain valuable information about $R / I$. For instance, since the Hilbert series is an additive function, we get the Hilbert series of $R / I$ from its Noether resolution. Moreover, whenever $I$ is a homogeneous ideal, i.e., homogeneous for the weight vector $\omega=(1, \ldots, 1)$, one can obtain the Castelnuovo-Mumford regularity of $R / I$ in terms of the Noether resolution as $\operatorname{reg}(R / I)=\max \left\{s_{i, v}-i \mid 0 \leq i \leq p\right.$, $\left.v \in \mathcal{B}_{i}\right\}$. 
In Section 2 we start by describing in Proposition 1 the first step of the Noether resolution of $R / I$. By Auslander-Buchsbaum formula, the depth of $R / I$ equals $d-p$. Hence, $R / I$ is CohenMacaulay if and only if $p=0$ or, equivalently, if $R / I$ is a free $A$-module. This observation together with Proposition 1, lead to Proposition 2 which is an effective criterion for determining whether $R / I$ is Cohen-Macaulay or not. This criterion generalizes [Bermejo \& Gimenez (2001), Proposition 2.1]. If $R / I$ is Cohen-Macaulay, Proposition 1 provides the whole Noether resolution of $R / I$. When $d=1$ and $R / I$ is not Cohen-Macaulay, we describe the Noether resolution of $R / I$ by means of Proposition 1 together with Proposition 3. Moreover, when $d=2$ and $x_{n}$ is a nonzero divisor of $R / I$, we are able to provide in Theorem 1 a complete description of the Noether resolution of $R / I$. All these results rely in the computation of a minimal Gröbner basis of $I$ with respect to the weighted degree reverse lexicographic order. As a consequence of this, we provide in Corollary 1 a description of the weighted Hilbert series in terms of the same Gröbner basis. Whenever $I$ is a homogeneous ideal, as a consequence of Theorem 11, we obtain in Corollary 2 a formula for the Castelnuovo-Mumford regularity of $R / I$ which is equivalent to the one provided in [Bermejo \& Gimenez (2000), Theorem 2.7].

In section 3 we study Noether resolutions when $R / I$ is a simplicial semigroup ring, i.e., whenever $I$ is a toric ideal and $A=K\left[x_{n-d+1}, \ldots, x_{n}\right]$ is a Noether normalization of $R / I$. We recall that $I$ is a toric ideal if $I=I_{\mathcal{A}}$ with $\mathcal{A}=\left\{a_{1}, \ldots, a_{n}\right\} \subset \mathbb{N}^{d}$ and $a_{i}=\left(a_{i 1}, \ldots, a_{i d}\right) \in \mathbb{N}^{d}$; where $I_{\mathcal{A}}$ denotes the kernel of the homomorphism of $K$-algebras $\varphi: R \rightarrow K\left[t_{1}, \ldots, t_{d}\right] ; x_{i} \mapsto t^{a_{i}}=$ $t_{1}^{a_{i 1}} \cdots t_{d}^{a_{i d}}$ for all $i \in\{1, \ldots, n\}$. If we denote by $\mathcal{S} \subset \mathbb{N}^{d}$ the semigroup generated by $a_{1}, \ldots, a_{n}$, then the image of $\varphi$ is $K[\mathcal{S}]:=K\left[t^{s} \mid s \in \mathcal{S}\right] \simeq R / I_{\mathcal{A}}$. By [Sturmfels (1996), Corollary 4.3], $I_{\mathcal{A}}$ is multigraded with respect to the grading induced by $\mathcal{S}$ which assigns $\operatorname{deg}_{\mathcal{S}}\left(x_{i}\right)=a_{i}$ for all $i \in\{1, \ldots, n\}$. Moreover, whenever $A$ is a Noether normalization of $K[\mathcal{S}]$ we may assume without loss of generality that $a_{n-d+i}=w_{n-d+i} e_{i}$ for all $i \in\{1, \ldots, d\}$, where $\omega_{n-d+i} \in \mathbb{Z}^{+}$and $\left\{e_{1}, \ldots, e_{d}\right\}$ is the canonical basis of $\mathbb{N}^{d}$. In this setting we may consider a multigraded Noether resolution of $K[\mathcal{S}]$, i.e., a minimal multigraded free resolution of $K[\mathcal{S}]$ as $A$-module:

$$
0 \longrightarrow \oplus_{s \in \mathcal{S}_{p}} A \cdot s \stackrel{\psi_{p}}{\longrightarrow} \cdots \stackrel{\psi_{1}}{\longrightarrow} \oplus_{s \in \mathcal{S}_{0}} A \cdot s \stackrel{\psi_{0}}{\longrightarrow} K[\mathcal{S}] \longrightarrow 0,
$$

where $\mathcal{S}_{i}$ are finite subsets of $\mathcal{S}$ for all $i \in\{0, \ldots, p\}$ and $A \cdot s$ denotes the shifting of $A$ by $s \in \mathcal{S}$. We observe that this multigrading is a refinement of the grading given by $\omega=\left(\omega_{1}, \ldots, \omega_{n}\right)$ with $\omega_{i}:=\sum_{j=1}^{d} a_{i j} \in \mathbb{Z}^{+}$; thus, $I_{\mathcal{A}}$ is weighted homogeneous with respect to $\omega$. As a consequence, whenever we get the multigraded Noether resolution or the multigraded Hilbert series of $K[\mathcal{S}]$, we also obtain its Noether resolution and its Hilbert series with respect to the weight vector $\omega$.

A natural and interesting problem is to describe combinatorially the multigraded Noether resolution of $K[\mathcal{S}]$ in terms of the semigroup $\mathcal{S}$. This approach would lead us to results for simplicial semigroup rings $K[\mathcal{S}]$ which do not depend on the characteristic of the field $K$. In general, for any toric ideal, it is well known that the minimal number of binomial generators of $I_{\mathcal{A}}$ does not depend on the characteristic of $K$ (see, e.g., [Sturmfels (1996), Theorem 5.3]), but the Gorenstein, Cohen-Macaulay and Buchsbaum properties of $K[\mathcal{S}]$ depend on the characteristic of $K$ (see [Hoa (1991)], [Trung \& Hoa (1986)] and [Hoa (1988)], respectively). However, in the context of simplicial semigroup rings, these properties do not depend on the characteristic of $K$ (see [Goto et al. (1976)], [Stanley (1978)] and [García-Sánchez \& Rosales (2002)], respectively). These facts give support to our aim of describing the whole multigraded Noether resolution of $K[\mathcal{S}]$ in terms of the underlying semigroup $\mathcal{S}$ for simplicial semigroup rings.

The results in section 3 are the following. In Proposition 5 we describe the first step of the multigraded Noether resolution of a simplicial semigroup ring $K[\mathcal{S}]$. As a byproduct we recover in Proposition 6 a well-known criterion for $K[\mathcal{S}]$ to be Cohen-Macaulay in terms of the semigroup. When $d=2$, i.e., $I_{\mathcal{A}}$ is the ideal of an affine toric surface, Theorem 2 describes the second step of the multigraded Noether resolution in terms of the semigroup $\mathcal{S}$. When $d=2$, from Proposition 5 and Theorem 2, we derive the whole multigraded Noether resolution of $K[\mathcal{S}]$ by means of $\mathcal{S}$ and, as a byproduct, we also get in Corollary 3 its multigraded Hilbert series. Whenever $I_{\mathcal{A}}$ a is 
homogeneous ideal, we get a formula for the Castelnuovo-Mumford regularity of $K[\mathcal{S}]$ in terms of $\mathcal{S}$, see Remark 1 .

Given an algebraic variety, the set of points where $X$ is not Cohen-Macaulay is the non CohenMacaulay locus. Macaulayfication is an analogous operation to resolution of singularities and was considered in Kawasaki [Kawasaki (2000)], where he provides certain sufficient conditions for $X$ to admit a Macaulayfication. For semigroup rings Goto et al. [Goto et al. (1976)] and Trung and Hoa [Trung \& Hoa (1986)] proved the existence of a semigroup $\mathcal{S}^{\prime}$ satisfying $\mathcal{S} \subset \mathcal{S}^{\prime} \subset \overline{\mathcal{S}}$, where $\overline{\mathcal{S}}$ denotes the saturation of $\mathcal{S}$ and thus $K[\overline{\mathcal{S}}]$ is the normalization of $K[\mathcal{S}]$, such that we have an exact sequence:

$$
0 \longrightarrow K[\mathcal{S}] \longrightarrow K\left[\mathcal{S}^{\prime}\right] \longrightarrow K\left[\mathcal{S}^{\prime} \backslash \mathcal{S}\right] \longrightarrow 0
$$

with $\operatorname{dim}\left(K\left[\mathcal{S}^{\prime} \backslash \mathcal{S}\right]\right) \leq \operatorname{dim}(K[\mathcal{S}])-2$. In this setting, $K\left[\mathcal{S}^{\prime}\right]$ satisfies the condition $S_{2}$ of Serre, and is called the $S_{2}$-fication of $K[\mathcal{S}]$. Moreover, when $\mathcal{S}$ is a simplicial semigroup, [Morales (2007), Theorem 5] proves that this semigroup ring $K\left[\mathcal{S}^{\prime}\right]$ is exactly the Macaulayfication of $K[\mathcal{S}]$; indeed, he proved that $K\left[\mathcal{S}^{\prime}\right]$ is Cohen-Macaulay and the support of $K\left[\mathcal{S}^{\prime} \backslash \mathcal{S}\right]$ coincides with the non Cohen-Macaulay locus of $K[\mathcal{S}]$. In [Morales (2007)], the author provides an explicit description of the Macaulayfication of $K[\mathcal{S}]$ in terms of the system of generators of $I_{\mathcal{A}}$ provided $K[\mathcal{S}]$ is a codimension 2 simplicial semigroup ring. Section 4 is devoted to study the Macaulayfication of any simplicial semigroup ring. The main result of this section is Theorem 4, where we entirely describe the Macaulayfication of any simplicial semigroup ring $K[\mathcal{S}]$ in terms of the set $\mathcal{S}_{0}$, the subset of $\mathcal{S}$ that provides the first step of the multigraded Noether resolution of $K[\mathcal{S}]$.

In sections 5 and 6 we apply the methods and results obtained in the previous ones to certain dimension 2 semigroup rings. More precisely, a sequence $m_{1}<\cdots<m_{n}$ determines the projective monomial curve $\mathcal{C} \subset \mathbb{P}_{K}^{n}$ parametrically defined by $x_{i}:=s^{m_{i}} t^{m_{n}-m_{i}}$ for all $i \in\{1, \ldots, n-1\}, x_{n}=s^{m_{n}}, x_{n+1}:=t^{m_{n}}$. If we set $\mathcal{A}=\left\{a_{1}, \ldots, a_{n+1}\right\} \subset \mathbb{N}^{2}$ where $a_{i}:=\left(m_{i}, m_{n}-m_{i}\right), a_{n}:=\left(m_{n}, 0\right)$ and $a_{n+1}:=\left(0, m_{n}\right)$, it turns out that the homogeneous coordinate ring of $\mathcal{C}$ is $K[\mathcal{C}]:=K\left[x_{1}, \ldots, x_{n+1}\right] / I_{\mathcal{A}}$ and $A=K\left[x_{n}, x_{n+1}\right]$ is a Noether normalization of $R / I_{\mathcal{A}}$.

The main result in Section 5 is Theorem [5, where we provide an upper bound on the CastelnuovoMumford regularity of $K[\mathcal{C}]$, where $\mathcal{C}$ is a projective monomial curve. The proof of this bound is elementary and builds on the results of the previous sections together with some classical results on numerical semigroups. It is known that $\operatorname{reg}(K[\mathcal{C}]) \leq m_{n}-n+1$ after the work [Gruson et al. (1983)]. In our case, [L'vovsky (1996)] obtained a better upper bound, indeed if we set $m_{0}:=0$ he proved that $\operatorname{reg}(\bar{K}[\mathcal{C}]) \leq \max _{1 \leq i<j \leq n}\left\{m_{i}-m_{i-1}+m_{j}-m_{j-1}\right\}-1$. The proof provided by L'vovsky is quite involved and uses advanced cohomological tools, it would be interesting to know if our results could yield a combinatorial alternative proof of this result. Even if L'vovsky's bound usually gives a better estimate than the bound we provide here, we easily construct families such that our bound outperforms the one by L'vovsky.

Also in the context of projective monomial curves, whenever $m_{1}<\cdots<m_{n}$ is an arithmetic sequence of relatively prime integers, the simplicial semigroup ring $R / I_{\mathcal{A}}$ has been extensively studied (see, e.g., [Molinelli \& Tamone (1995), Li et. al (2012), Bermejo et al. (2017)]) and the multigraded Noether resolution is easy to obtain. In Section 6, we study the coordinate ring of the canonical projections of projective monomial curves associated to arithmetic sequences, i.e., the curves $\mathcal{C}_{r}$ whose homogeneous coordinate rings are $K\left[\mathcal{S}_{r}\right]=R / I_{\mathcal{A}_{r}}$, where $\mathcal{A}_{r}:=\mathcal{A} \backslash\left\{a_{r}\right\}$ and $\mathcal{S}_{r} \subset \mathbb{N}^{2}$ the semigroup generated by $\mathcal{A}_{r}$ for all $r \in\{1, \ldots, n-1\}$. In Corollary 5 we give a criterion for determining when the semigroup ring $K\left[\mathcal{S}_{r}\right]$ is Cohen-Macaulay; whenever it is not Cohen-Macaulay, we get its Macaulayfication in Corollary 6 . Furthermore, in Theorem 7 we provide an explicit description of their multigraded Noether resolutions. Finally, in Theorem 8 we get a formula for their Castelnuovo-Mumford regularity. 


\section{NOETHER RESOlution. GENERAL CASE}

Let $R:=K\left[x_{1}, \ldots, x_{n}\right]$ be a polynomial ring over an infinite field $K$, and let $I \subset R$ be a $\omega$-homogeneous ideal, i.e., a weighted homogeneous ideal with respect to the vector $\omega=$ $\left(\omega_{1}, \ldots, \omega_{n}\right) \in\left(\mathbb{Z}^{+}\right)^{n}$. We assume that $A:=K\left[x_{n-d+1}, \ldots, x_{n}\right]$ is a Noether normalization of $R / I$, where $d:=\operatorname{dim}(R / I)$. In this section we study the Noether resolution of $R / I$, i.e., the minimal graded free resolution of $R / I$ as $A$-module:

$$
\mathcal{F}: 0 \longrightarrow \oplus_{v \in \mathcal{B}_{p}} A\left(-s_{p, v}\right) \stackrel{\psi_{p}}{\longrightarrow} \cdots \stackrel{\psi_{1}}{\longrightarrow} \oplus_{v \in \mathcal{B}_{0}} A\left(-s_{0, v}\right) \stackrel{\psi_{0}}{\longrightarrow} R / I \longrightarrow 0,
$$

where for all $i \in\{0, \ldots, p\} \mathcal{B}_{i}$ is a finite set of monomials, and $s_{i, v}$ are nonnegative integers.

In order to obtain the first step of the resolution, we will deal with the initial ideal of $I+$ $\left(x_{n-d+1}, \ldots, x_{n}\right)$ with respect to the weighted degree reverse lexicographic order $>_{\omega}$.

We recall that $>_{\omega}$ is defined as follows: $x^{\alpha}>_{\omega} x^{\beta}$ if and only if

- $\operatorname{deg}_{\omega}\left(x^{\alpha}\right)>\operatorname{deg}_{\omega}\left(x^{\beta}\right)$, or

- $\operatorname{deg}_{\omega}\left(x^{\alpha}\right)=\operatorname{deg}_{\omega}\left(x^{\beta}\right)$ and the last nonzero entry of $\alpha-\beta \in \mathbb{Z}^{n}$ is negative.

For every polynomial $f \in R$ we denote by in $(f)$ the initial term of $f$ with respect to $>_{\omega}$. Analogously, for every ideal $J \subset R$, in $(J)$ denotes its initial ideal with respect to $>_{\omega}$.

Proposition 1. Let $\mathcal{B}_{0}$ be the set of monomials that do not belong to in $\left(I+\left(x_{n-d+1}, \ldots, x_{n}\right)\right)$ Then,

$$
\left\{x^{\alpha}+I \mid x^{\alpha} \in \mathcal{B}_{0}\right\}
$$

is a minimal set of generators of $R / I$ as A-module and the shifts of the first step of the Noether resolution (1) are given by $\operatorname{deg}_{\omega}\left(x^{\alpha}\right)$ with $x^{\alpha} \in \mathcal{B}_{0}$.

Proof. Since $A$ is a Noether normalization of $R / I$ we have that $\mathcal{B}_{0}$ is a finite set. Let $\mathcal{B}_{0}=$ $\left\{x^{\alpha_{1}}, \ldots, x^{\alpha_{k}}\right\}$. To prove that $\mathcal{B}:=\left\{x^{\alpha_{1}}+I, \ldots, x^{\alpha_{k}}+I\right\}$ is a set of generators of $R / I$ as $A$-module it suffices to show that for every monomial $x^{\beta}:=x_{1}^{\beta_{1}} \cdots x_{n-d}^{\beta_{n-d}} \notin$ in $(I)$, one has that $x^{\beta}+I \in R / I$ can be written as a linear combination of $\left\{x^{\alpha_{1}}+I, \ldots, x^{\alpha_{k}}+I\right\}$. Since $\left\{x^{\alpha_{1}}+(I+\right.$ $\left.\left.\left(x_{n-d+1}, \ldots, x_{n}\right)\right), \ldots, x^{\alpha_{k}}+\left(I+\left(x_{n-d+1}, \ldots, x_{n}\right)\right)\right\}$ is a $K$-basis of $R /\left(I+\left(x_{n-d+1}, \ldots, x_{n}\right)\right)$, we have that $g:=x^{\beta}-\sum_{i=1}^{k} \lambda_{i} x^{\alpha_{i}} \in I+\left(x_{n-d+1}, \ldots, x_{n}\right)$ for some $\lambda_{1}, \ldots, \lambda_{k} \in K$. Then we deduce that in $(g) \in$ in $\left(I+\left(x_{n-d+1}, \ldots, x_{n}\right)\right)$ which is equal to in $(I)+\left(x_{n-d+1}, \ldots, x_{n}\right)$, and thus in $(g) \in$ in $(I)$. Since $x^{\beta} \notin$ in $(I)$ and $x^{\alpha_{i}} \notin$ in $(I)$ for all $i \in\{1, \ldots, k\}$, we conclude that $g=0$ and $x^{\beta}+I=\left(\sum_{i=1}^{k} \lambda_{i} x^{\alpha_{i}}\right)+I$. The minimality of $\mathcal{B}$ can be easily proved.

When $R / I$ is a free $A$-module or, equivalently, when the projective dimension of $R / I$ as $A$ module is 0 and hence $R / I$ is Cohen-Macaulay, Proposition 1 provides the whole Noether resolution of $R / I$. In Proposition 2 we characterize the Cohen-Macaulay property for $R / I$ in terms of the initial ideal in $(I)$ previously defined. This result generalizes [Bermejo \& Gimenez (2001), Theorem 2.1], which applies for $I$ a homogeneous ideal.

Proposition 2. Let $A=K\left[x_{n-d+1}, \ldots, x_{n}\right]$ be a Noether normalization of $R / I$. Then, $R / I$ is Cohen-Macaulay if and only if $x_{n-d+1}, \ldots, x_{n}$ do not divide any minimal generator of in $(I)$.

Proof. We denote by $\left\{e_{v} \mid v\right.$ in $\left.\mathcal{B}_{0}\right\}$ the canonical basis of $\oplus_{v \in \mathcal{B}_{0}} A\left(-\operatorname{deg}_{\omega}(v)\right)$. By Proposition 1 we know that $\psi_{0}: \oplus_{v \in \mathcal{B}_{0}} A\left(-\operatorname{deg}_{\omega}(v)\right) \longrightarrow R / I$ is the morphism induced by $e_{v} \mapsto v+I \in R / I$. By Auslander-Buchsbaum formula, $R / I$ is Cohen-Macaulay if and only if $\psi_{0}$ is injective.

$(\Rightarrow)$ By contradiction, we assume that there exists $\alpha=\left(\alpha_{1}, \ldots, \alpha_{n}\right) \in \mathbb{N}^{n}$ such that $x^{\alpha}=$ $x_{1}^{\alpha_{1}} \cdots x_{n}^{\alpha_{n}}$ is a minimal generator of in $(I)$ and that $\alpha_{i}>0$ for some $i \in\{n-d+1, \ldots, n\}$. Set $u:=x_{1}^{\alpha_{1}} \cdots x_{n-d}^{\alpha_{n-d}}$, since $\operatorname{in}\left(I+\left(x_{n-d+1}, \ldots, x_{n}\right)\right)=\operatorname{in}(I)+\left(x_{n-d+1}, \ldots, x_{n}\right)$, we have that $u \in \mathcal{B}_{0}$. We also set $x^{\alpha^{\prime}}:=x_{n-d+1}^{\alpha_{n-d+1}} \cdots x_{n}^{\alpha_{n}} \in A$ and $f$ the remainder of $x^{\alpha}$ modulo the reduced Gröbner basis of $I$ with respect to $>_{\omega}$. Then $x^{\alpha}-f \in I$ and every monomial in $f$ does not belong to in $(I)$. As a consequence, $f=\sum_{i=1}^{t} c_{i} x^{\beta_{i}}$, where $c_{i} \in K$ and $x^{\beta_{i}}=v_{i} x^{\beta_{i}^{\prime}}$ with $v_{i} \in \mathcal{B}_{0}$ 
and $x^{\beta_{i}^{\prime}} \in A$ for all $i \in\{1, \ldots, t\}$. Hence, $x^{\alpha^{\prime}} e_{u}-\sum_{i=1}^{t} c_{i} x^{\beta_{i}^{\prime}} e_{v_{i}} \in \operatorname{Ker}\left(\psi_{0}\right)$ and $R / I$ is not Cohen-Macaulay.

$(\Leftarrow)$ Assume that there exists a nonzero $g \in \operatorname{Ker}\left(\psi_{0}\right)$, namely, $g=\sum_{v \in \mathcal{B}_{0}} g_{v} e_{v} \in \operatorname{Ker}\left(\psi_{0}\right)$ with $g_{v} \in A$ for all $v \in \mathcal{B}_{0}$. Then, $\sum_{v \in \mathcal{B}_{0}} g_{v} v \in I$. We write in $(g)=c x^{\alpha} u$ with $c \in K, x^{\alpha} \in A$ and $u \in \mathcal{B}_{0}$. Since $x_{n-d+1}, \ldots, x_{n}$ do not divide any minimal generator of in $(I)$, we have that $u \in$ in $(I)$, a contradiction.

When $R / I$ has dimension 1 , its depth can be either 0 or 1 . When $\operatorname{depth}(R / I)=1$, then $R / I$ is Cohen-Macaulay and the whole Noether resolution is given by Proposition 1 . When $R / I$ is not Cohen-Macaulay, then its depth is 0 and its projective dimension as $A$-module is 1 . In this setting, to describe the whole Noether resolution it remains to determine $\mathcal{B}_{1}, \psi_{1}$ and the shifts $s_{1, v} \in \mathbb{N}$ for all $v \in \mathcal{B}_{1}$. In Proposition 3 we explain how to obtain $\mathcal{B}_{1}$ and $\psi_{1}$ by means of a Gröbner basis of $I$ with respect to $>\omega$.

Consider $\chi_{1}: R \longrightarrow R$ the evaluation morphism induced by $x_{i} \mapsto x_{i}$ for $i \in\{1, \ldots, n-1\}$, $x_{n} \mapsto 1$.

Proposition 3. Let $R / I$ be 1-dimensional ring of depth 0 . Let $L$ be the ideal $\chi_{1}(\operatorname{in}(I)) \cdot R$. Then,

$$
\mathcal{B}_{1}=\mathcal{B}_{0} \cap L
$$

in the Noether resolution (1) of $R / I$ and the shifts of the second step of this resolution are given by $\operatorname{deg}_{\omega}\left(u x_{n}^{\delta_{u}}\right)$, where $u \in \mathcal{B}_{1}$ and $\delta_{u}:=\min \left\{\delta \mid u x_{n}^{\delta} \in \operatorname{in}(I)\right\}$.

Proof. For every $u=x_{1}^{\alpha_{1}} \cdots x_{n-1}^{\alpha_{n-1}} \in \mathcal{B}_{0} \cap L$, there exists $\delta \in \mathbb{N}$ such that $u x_{n}^{\delta} \in \operatorname{in}(I)$; let $\delta_{u}$ be the minimum of all such $\delta$. Consider $p_{u} \in R$ the remainder of $u x_{n}^{\delta_{u}}$ modulo the reduced Gröbner basis of $I$ with respect to $>_{\omega}$. Thus $u x_{n}^{\delta_{u}}-p_{u} \in I$ is $\omega$-homogeneous and every monomial $x^{\beta}$ appearing in $p_{u}$ does not belong to in $(I)$, then by Proposition 1 it can be expressed as $x^{\beta}=v x_{n}^{\beta_{n}}$, where $\beta_{n} \geq 0$ and $v \in \mathcal{B}_{0}$. Moreover, since $u x_{n}^{\delta_{u}}>_{\omega} x^{\beta}$, then $\beta_{n} \geq \delta_{u}$ and $u>_{\omega} v$. Thus, we can write

$$
p_{u}=\sum_{\substack{v \in \mathcal{B}_{0} \\ u>\omega v}} x_{n}^{\delta_{u}} m_{u_{v}} v
$$

with $m_{u_{v}}=c x^{\alpha_{u_{v}}} \in A=K\left[x_{n}\right]$ a monomial (possibly 0) for all $v \in \mathcal{B}_{0}, u>_{\omega} v$.

Now we denote by $\left\{e_{v} \mid v\right.$ in $\left.\mathcal{B}_{0}\right\}$ the canonical basis of $\oplus_{v \in \mathcal{B}_{0}} A\left(-\operatorname{deg}_{\omega}(v)\right)$ and consider the graded morphism $\psi_{0}: \oplus_{v \in \mathcal{B}_{0}} A\left(-\operatorname{deg}_{\omega}(v)\right) \longrightarrow R / I$ induced by $e_{v} \mapsto v+I \in R / I$. The above construction yields that

$$
h_{u}:=x_{n}^{\delta_{u}}\left(e_{u}-\sum_{\substack{v \in \mathcal{B}_{0} \\ u>\omega v}} m_{u_{v}} e_{v}\right) \in \operatorname{Ker}\left(\psi_{0}\right)
$$

for all $u \in \mathcal{B}_{0} \cap L$. We will prove that $\operatorname{Ker}\left(\psi_{0}\right)$ is a free $A$-module with basis

$$
\mathcal{C}:=\left\{h_{u} \mid u \in \mathcal{B}_{0} \cap L\right\} .
$$

Firstly, we observe that the $A$-module generated by the elements of $\mathcal{C}$ is free due to the triangular form of the matrix formed by the elements of $\mathcal{C}$. Let us now take $g=\sum_{v \in \mathcal{B}_{0}} g_{v} e_{v} \in \operatorname{Ker}\left(\psi_{0}\right)$ with $g_{v} \in A$, we assume that $g \in \oplus_{v \in \mathcal{B}_{0}} A\left(-\operatorname{deg}_{\omega}(v)\right)$ is $\omega$-homogeneous and, thus, $g_{v}$ is either 0 or a monomial of the form $c x_{n}^{\beta_{v}}$ with $c \in K$ and $\beta_{v} \in \mathbb{N}$ for all $v \in \mathcal{B}_{0}$. We consider $\bar{\psi}_{0}$ : $\oplus_{v \in \mathcal{B}_{0}} A\left(-\operatorname{deg}_{\omega}(v)\right) \longrightarrow R$ the monomorphism of $A$-modules induced by $e_{v} \mapsto v$. Since $\psi_{0}(g)=$ 0 , then the polynomial $g^{\prime}:=\bar{\psi}_{0}(g)=\sum_{u \in \mathcal{B}_{0}} g_{u} u \in I$ and $\operatorname{in}\left(g^{\prime}\right)=c x_{n}^{\gamma} w$ for some $w \in \mathcal{B}_{0}$, $\gamma \in \mathbb{N}$ and $c \in K$. Since in $\left(g^{\prime}\right) \in \operatorname{in}(I)$, we get that $w \in \mathcal{B}_{0} \cap L$ and $\gamma \geq \delta_{w}$. Hence, $g_{1}:=g-c x_{n-1}^{\gamma-\delta_{w}} h_{w} \in \operatorname{Ker}\left(\psi_{0}\right)$. If $g_{1}$ is identically zero, then $g \in\left(\left\{h_{u} \mid u \in \mathcal{B}_{0} \cap L\right\}\right)$. If $g_{1}$ is not zero, we have that $0 \neq \operatorname{in}\left(\bar{\psi}_{0}\left(g_{1}\right)\right)<\operatorname{in}\left(\bar{\psi}_{0}(g)\right)$ and we iterate this process with $g_{1}$ to derive that $\left\{h_{u} \mid u \in \mathcal{B}_{0} \cap L\right\}$ generates $\operatorname{Ker}\left(\psi_{0}\right)$. 
The rest of this section concerns $I$ a saturated ideal such that $R / I$ is 2-dimensional and it is not Cohen-Macaulay (and, in particular, $\operatorname{depth}(R / I)=1$ ). We assume that $A=K\left[x_{n-1}, x_{n}\right]$ is a Noether normalization of $R / I$ and we aim at describing the whole Noether resolution of $R / I$. To achieve this it only remains to describe $\mathcal{B}_{1}, \psi_{1}$ and the shifts $s_{1, v} \in \mathbb{N}$ for all $v \in \mathcal{B}_{1}$. In Proposition 4 we explain how to obtain $\mathcal{B}_{1}$ and $\psi_{1}$ by means of a Gröbner basis of $I$ with respect to $>_{\omega}$. Since $K$ is an infinite field, $I$ is a saturated ideal and $A$ is a Noether normalization of $R / I$, one has that $x_{n}+\tau x_{n-1}$ is a nonzero divisor on $R / I$ for all $\tau \in K$ but a finite set. Thus, by performing a mild change of coordinates if necessary, we may assume that $x_{n}$ is a nonzero divisor on $R / I$.

Now consider $\chi: R \longrightarrow R$ the evaluation morphism induced by $x_{i} \mapsto x_{i}$ for $i \in\{1, \ldots, n-2\}$, $x_{i} \mapsto 1$ for $i \in\{n-1, n\}$.

Proposition 4. Let $R / I$ be 2-dimensional, non Cohen-Macaulay ring such that $x_{n}$ is a nonzero divisor. Let $J$ be the ideal $\chi(\operatorname{in}(I)) \cdot R$. Then,

$$
\mathcal{B}_{1}=\mathcal{B}_{0} \cap J
$$

in the Noether resolution (1) of $R / I$ and the shifts of the second step of this resolution are given by $\operatorname{deg}_{\omega}\left(u x_{n-1}^{\delta_{u}}\right)$, where $u \in \mathcal{B}_{1}$ and $\delta_{u}:=\min \left\{\delta \mid u x_{n-1}^{\delta} \in \operatorname{in}(I)\right\}$.

Proof. Since $x_{n}$ is a nonzero divisor of $R / I$ and $I$ is a $\omega$-homogeneous ideal, then $x_{n}$ does not divide any minimal generator of in $(I)$. As a consequence, for every $u=x_{1}^{\alpha_{1}} \cdots x_{n-2}^{\alpha_{n-2}} \in \mathcal{B}_{0} \cap J$, there exists $\delta \in \mathbb{N}$ such that $u x_{n-1}^{\delta} \in \operatorname{in}(I)$; by definition, $\delta_{u}$ is the minimum of all such $\delta$. Consider $p_{u} \in R$ the remainder of $u x_{n-1}^{\delta_{u}}$ modulo the reduced Gröbner basis of $I$ with respect to $>_{\omega}$. Then $u x_{n-1}^{\delta_{u}}-p_{u} \in I$ is $\omega$-homogeneous and every monomial $x^{\beta}$ appearing in $p_{u}$ does not belong to in $(I)$, then by Proposition 1 it can be expressed as $x^{\beta}=v x_{n-1}^{\beta_{n-1}} x_{n}^{\beta_{n}}$, where $\beta_{n-1}, \beta_{n} \geq 0$ and $v \in \mathcal{B}_{0}$. Moreover, we have that $u x_{n-1}^{\delta_{u}}>_{\omega} x^{\beta}$ which implies that either $\beta_{n} \geq 1$, or $\beta_{n}=0$, $\beta_{n-1} \geq \delta_{u}$ and $u>_{\omega} v$. Thus, we can write

$$
p_{u}=\sum_{\substack{v \in \mathcal{B}_{0} \\ u>\omega v}} x_{n-1}^{\delta_{u}} f_{u_{v}} v+\sum_{v \in \mathcal{B}_{0}} x_{n} g_{u_{v}} v,
$$

with $f_{u_{v}} \in K\left[x_{n-1}\right]$ for all $v \in \mathcal{B}_{0}, u>_{\omega} v$ and $g_{u_{v}} \in A$ for all $v \in \mathcal{B}_{0}$.

Now we denote by $\left\{e_{v} \mid v\right.$ in $\left.\mathcal{B}_{0}\right\}$ the canonical basis of $\oplus_{v \in \mathcal{B}_{0}} A\left(-\operatorname{deg}_{\omega}(v)\right)$ and consider the graded morphism $\psi_{0}: \oplus_{v \in \mathcal{B}_{0}} A\left(-\operatorname{deg}_{\omega}(v)\right) \longrightarrow R / I$ induced by $e_{v} \mapsto v+I \in R / I$. The above construction yields that

$$
h_{u}:=x_{n-1}^{\delta_{u}} e_{u}-\sum_{\substack{v \in \mathcal{B}_{0} \\ u>\omega_{v}}} x_{n-1}^{\delta_{u}} f_{u_{v}} e_{v}-\sum_{v \in \mathcal{B}_{0}} x_{n} g_{u_{v}} e_{v} \in \operatorname{Ker}\left(\psi_{0}\right)
$$

for all $u \in \mathcal{B}_{0} \cap J$. We will prove that $\operatorname{Ker}\left(\psi_{0}\right)$ is a free $A$-module with basis

$$
\mathcal{C}:=\left\{h_{u} \mid u \in \mathcal{B}_{0} \cap J\right\} \text {. }
$$

Firstly, we prove that the $A$-module generated by the elements of $\mathcal{C}$ is free. Assume that $\sum_{u \in \mathcal{B}_{0} \cap J} q_{u} h_{u}=0$ where $q_{u} \in A$ for all $u \in \mathcal{B}_{0} \cap J$ and we may also assume that $x_{n}$ does not divide $q_{v}$ for some $v \in \mathcal{B}_{0} \cap J$. We consider the evaluation morphism $\tau$ induced by $x_{n} \mapsto 0$ and we get that $\sum_{u \in \mathcal{B}_{0} \cap J} \tau\left(q_{u}\right) \tau\left(h_{u}\right)=\sum_{u \in \mathcal{B}_{0} \cap J} \tau\left(q_{u}\right)\left(x_{n-1}^{\delta_{u}} e_{u}+\sum_{\substack{v \in \mathcal{B}_{0} \\ u>\omega v}} x_{n-1}^{\delta_{u}} f_{u_{v}} e_{v}\right)=0$, which implies that $\tau\left(q_{u}\right)=0$ for all $u \in \mathcal{B}_{0} \cap J$ and, hence, $x_{n} \mid q_{u}$ for all $u \in \mathcal{B}_{0} \cap J$, a contradiction.

Let us take $g=\sum_{v \in \mathcal{B}_{0}} g_{v} e_{v} \in \operatorname{Ker}\left(\psi_{0}\right)$ with $g_{v} \in A$, we assume that $g \in \oplus_{v \in \mathcal{B}_{0}} A\left(-\operatorname{deg}_{\omega}(v)\right)$ is $\omega$-homogeneous and, thus, $g_{v}$ is either 0 or a $\omega$-homogeneous polynomial for all $v \in \mathcal{B}_{0}$. We may also suppose that there exists $v \in \mathcal{B}_{0}$ such that $x_{n}$ does not divide $g_{v}$. We consider $\bar{\psi}_{0}: \oplus_{v \in \mathcal{B}_{0}} A\left(-\operatorname{deg}_{\omega}(v)\right) \longrightarrow R$ the monomorphism of $A$-modules induced by $e_{v} \mapsto v$. Since $\psi_{0}(g)=0$, then the polynomial $g^{\prime}:=\bar{\psi}_{0}(g)=\sum_{u \in \mathcal{B}_{0}} g_{u} u \in I$ and $\operatorname{in}\left(g^{\prime}\right)=c x_{n-1}^{\gamma} w$ for some $w \in \mathcal{B}_{0}$ and some $c \in K$, which implies that $w \in \mathcal{B}_{0} \cap J$. By definition of $\delta_{w}$ we get that $\gamma \geq \delta_{w}$, hence $g_{1}:=g-c x_{n-1}^{\gamma-\delta_{w}} h_{w} \in \operatorname{Ker}\left(\psi_{0}\right)$. If $g_{1}$ is identically zero, then $g \in\left(\left\{h_{u} \mid u \in \mathcal{B}_{0} \cap J\right\}\right)$. 
If $g_{1}$ is not zero, we have that $0 \neq \operatorname{in}\left(\bar{\psi}_{0}\left(g_{1}\right)\right)<\operatorname{in}\left(\bar{\psi}_{0}(g)\right)$ and we iterate this process with $g_{1}$ to derive that $\left\{h_{u} \mid u \in \mathcal{B}_{0} \cap J\right\}$ generates $\operatorname{Ker}\left(\psi_{0}\right)$.

From Propositions 1 and 4 and their proofs, we can obtain the Noether resolution $\mathcal{F}$ of $R / I$ by means of a Gröbner basis of $I$ with respect to $>_{\omega}$. We also observe that for obtaining the shifts of the resolution it suffices to know a set of generators of in $(I)$. The following theorem gives the resolution.

Theorem 1. Let $R / I$ be a 2-dimensional ring such that $x_{n}$ is a nonzero divisor. We denote by $\mathcal{G}$ be a Gröbner basis of I with respect to $>_{\omega}$. If $\delta_{u}:=\min \left\{\delta \mid u x_{n-1}^{\delta} \in \operatorname{in}(I)\right\}$ for all $u \in \mathcal{B}_{1}$, then

$$
\mathcal{F}: 0 \longrightarrow \oplus_{u \in \mathcal{B}_{1}} A\left(-\operatorname{deg}_{\omega}(u)-\delta_{u} \omega_{n-1}\right) \stackrel{\psi_{1}}{\longrightarrow} \oplus_{v \in \mathcal{B}_{0}} A\left(-\operatorname{deg}_{\omega}(v)\right) \stackrel{\psi_{0}}{\longrightarrow} R / I \longrightarrow 0,
$$

is the Noether resolution of $R / I$, where

$$
\begin{aligned}
\psi_{0}: \oplus_{v \in \mathcal{B}_{0}} A\left(-\operatorname{deg}_{\omega}(v)\right) & \rightarrow R / I, \\
e_{v} & \mapsto v+I
\end{aligned}
$$

and

$$
\begin{aligned}
\psi_{1}: \oplus_{u \in \mathcal{B}_{1}} A\left(-\operatorname{deg}_{\omega}(u)-\delta_{u} \omega_{n-1}\right) & \longrightarrow \oplus_{v \in \mathcal{B}_{0}} A\left(-\operatorname{deg}_{\omega}(v)\right), \\
e_{u} & \mapsto x_{n-1}^{\delta_{u}} e_{u}-\sum_{v \in \mathcal{B}_{0}} f_{u_{v}} e_{v}
\end{aligned}
$$

whenever $\sum_{v \in \mathcal{B}_{0}} f_{u_{v}} v$ with $f_{u_{v}} \in A$ is the remainder of the division of $u x_{n-1}^{\delta_{u}}$ by $\mathcal{G}$.

From this resolution, we can easily describe the weighted Hilbert series of $R / I$.

Corollary 1. Let $R / I$ be a 2-dimensional ring such that $x_{n}$ is a nonzero divisor, then its Hilbert series is given by:

$$
H S_{R / I}(t)=\frac{\sum_{v \in \mathcal{B}_{0}} t^{\operatorname{deg}_{\omega}(v)}-\sum_{u \in \mathcal{B}_{1}} t^{\operatorname{deg}_{\omega}(u)+\delta_{u} w_{n-1}}}{\left(1-t^{\omega_{n-1}}\right)\left(1-t^{\omega_{n}}\right)}
$$

In the following example we show how to compute the Noether resolution and the weighted Hilbert series of the graded coordinate ring of a surface in $\mathbb{A}_{K}^{4}$.

Example 1. Let $I$ be the defining ideal of the surface of $\mathbb{A}_{K}^{4}$ parametrically defined by $f_{1}:=s^{3}+$ $s^{2} t, f_{2}:=t^{4}+s t^{3}, f_{3}:=s^{2}, f_{4}:=t^{2} \in K[s, t]$. Using Singular [Decker et al. (2015)], CoCoA [Abbott et al. (2015)] or MACAULAY 2 [Grayson \& Stillman (2015)] we obtain that whenever $K$ is a characteristic 0 field, the polynomials $\left\{g_{1}, g_{2}, g_{3}, g_{4}\right\}$ constitute a minimal Gröbner basis of its defining ideal with respect to $>_{\omega}$ with $\omega=(3,4,2,2)$, where $g_{1}:=2 x_{2} x_{3}^{2}-x_{1}^{2} x_{4}+x_{3}^{3} x_{4}-$ $x_{3}^{2} x_{4}^{2}, g_{2}:=x_{1}^{4}-2 x_{1}^{2} x_{3}^{3}+x_{3}^{6}-2 x_{1}^{2} x_{3}^{2} x_{4}-2 x_{3}^{5} x_{4}+x_{3}^{4} x_{4}^{2}, g_{3}:=x_{2}^{2}-2 x_{2} x_{4}^{2}-x_{3} x_{4}^{3}+x_{4}^{4}$ and $g_{4}:=2 x_{1}^{2} x_{2}-x_{1}^{2} x_{3} x_{4}+x_{3}^{4} x_{4}-3 x_{1}^{2} x_{4}^{2}-2 x_{3}^{3} x_{4}^{2}+x_{3}^{2} x_{4}^{3}$. In particular,

$$
\text { in }(I)=\left(x_{2} x_{3}^{2}, x_{1}^{4}, x_{2}^{2}, x_{1}^{2} x_{2}\right) \text {. }
$$

Then, we obtain that

- $\mathcal{B}_{0}=\left\{u_{1}, \ldots, u_{6}\right\}$ with $u_{1}:=1, u_{2}:=x_{1}, u_{3}:=x_{2}, u_{4}:=x_{1}^{2}, u_{5}:=x_{1} x_{2}, u_{6}:=x_{1}^{3}$,

- $J=\left(x_{2}, x_{1}^{4}\right) \subset K\left[x_{1}, x_{2}, x_{3}, x_{4}\right]$, and

- $\mathcal{B}_{1}=\left\{u_{3}\right\}$.

Since $x_{3}$ divides a minimal generator of in $(I)$, by Proposition 2 we deduce that $R / I$ is not CohenMacaulay. We compute $\delta_{3}=\min \left\{\delta \mid u_{3} x_{3}^{\delta} \in \operatorname{in}(I)\right\}$ and get that $\delta_{3}=2$ and that $r_{3}=-x_{4} u_{4}+$ $\left(x_{3}^{3} x_{4}-x_{3}^{2} x_{4}^{2}\right) u_{1}$ is the remainder of the division of $u_{3} x_{3}^{2}$ by $\mathcal{G}$. Hence, following Theorem $\square$ we obtain the Noether resolution or $R / I$ :

$$
\mathcal{F}: 0 \rightarrow A(-8) \stackrel{\psi}{\rightarrow} \begin{gathered}
A \oplus A(-3) \oplus A(-4) \oplus \\
\oplus A(-6) \oplus A(-7) \oplus A(-9)
\end{gathered} \rightarrow R / I \rightarrow 0
$$


where $\psi$ is given by the matrix

$$
\left(\begin{array}{c}
-x_{3}^{3} x_{4}+x_{3}^{2} x_{4}^{2} \\
0 \\
x_{3}^{2} \\
x_{4} \\
0 \\
0
\end{array}\right)
$$

Moreover, by Corollary 1 we obtain that the weighted Hilbert series of $R / I$ is

$$
H S_{R / I}(t)=\frac{1+t^{3}+t^{4}+t^{6}+t^{7}-t^{8}+t^{9}}{\left(1-t^{2}\right)^{2}} .
$$

If we consider the same parametric surface over an infinite field of characteristic 2 , we obtain that $\left\{x_{1}^{2}+x_{3}^{3}+x_{3}^{2} x_{4}, x_{2}^{2}+x_{3} x_{4}^{3}+x_{4}^{4}\right\}$ is a minimal Gröbner basis of I with respect to $>_{\omega}$, the weighted degree reverse lexicographic order with $\omega=(3,4,2,2)$. Then we have that

$$
\mathcal{B}_{0}=\left\{v_{1}:=1, v_{2}:=x_{1}, v_{3}:=x_{2}, v_{4}:=x_{1} x_{2}\right\},
$$

and $\mathcal{B}_{1}=\emptyset$, so $R / I$ is Cohen-Macaulay. Moreover, we also obtain the Noether resolution of $R / I$

$$
\mathcal{F}^{\prime}: 0 \rightarrow A \oplus A(-3) \oplus A(-4) \oplus A(-7) \rightarrow R / I \rightarrow 0
$$

and the weighted Hilbert series of $R / I$ is

$$
H S_{R / I}(t)=\frac{1+t^{3}+t^{4}+t^{7}}{\left(1-t^{2}\right)^{2}}
$$

To end this section, we consider the particular case where $I$ is standard graded homogeneous, i.e., $\omega=(1, \ldots, 1)$. In this setting, we obtain a formula for the Castelnuovo-Mumford regularity of $R / I$ in terms of in $(I)$ or, more precisely, in terms of $\mathcal{B}_{0}$ and $\mathcal{B}_{1}$. This formula is equivalent to that of [Bermejo \& Gimenez (2000), Theorem 2.7] provided $x_{n}$ is a nonzero divisor of $R / I$.

Corollary 2. Let $R / I$ be a 2-dimensional standard graded ring such that $x_{n}$ is a nonzero divisor. Then,

$$
\operatorname{reg}(R / I)=\max \left\{\operatorname{deg}(v), \operatorname{deg}(u)+\delta_{u}-1 \mid v \in \mathcal{B}_{0}, u \in \mathcal{B}_{1}\right\}
$$

In the following example we apply all the results of this section.

Example 2. Let $K$ be a characteristic zero field and let us consider the projective curve $\mathcal{C}$ of $\mathbb{P}_{K}^{4}$ parametrically defined by:

$$
x_{1}=s^{3} t^{5}-s t^{7}, x_{2}=s^{7} t, x_{3}=s^{4} t^{4}, x_{4}=s^{8}, x_{5}=t^{8} .
$$

A direct computation with SINGULAR, COCOA or MACAULAY 2 yields that a minimal Gröbner basis $\mathcal{G}$ of the defining ideal $I \subset R=K\left[x_{1}, \ldots, x_{5}\right]$ of $\mathcal{C}$ with respect to the degree reverse lexicographic order consists of 10 elements and that

$$
\text { in }(I)=\left(x_{1}^{4}, x_{2}^{4}, x_{1}^{3} x_{3}, x_{1} x_{3} x_{4}^{2}, x_{1}^{2} x_{2}, x_{1} x_{2}^{2}, x_{1} x_{2} x_{3}, x_{2}^{2} x_{3}, x_{1}^{2} x_{4}, x_{3}^{2}\right) .
$$

Then, we obtain that the set $\mathcal{B}_{0}$ is the following

$$
\begin{gathered}
\mathcal{B}_{0}=\left\{u_{1}:=1, u_{2}:=x_{1}, u_{3}:=x_{2}, u_{4}:=x_{3}, u_{5}:=x_{1}^{2}, u_{6}:=x_{1} x_{2}, u_{7}:=x_{2}^{2},\right. \\
\left.u_{8}:=x_{1} x_{3}, u_{9}:=x_{2} x_{3}, u_{10}:=x_{1}^{3}, u_{11}:=x_{2}^{3}, u_{12}:=x_{1}^{2} x_{3}\right\}
\end{gathered}
$$

and the ideal $J$ is

$$
J=\left(x_{1}^{2}, x_{1} x_{3}, x_{3}^{2}, x_{2}^{2} x_{3}, x_{2}^{4}\right) \subset R .
$$

Thus, $\mathcal{B}_{1}=\left\{u_{5}, u_{8}, u_{10}, u_{12}\right\}$. For $i \in\{5,8,10,12\}$ we compute $\delta_{i}$, the minimum integer such that $u_{i} x_{4}^{\delta_{i}} \in$ in $(I)$ and get that $\delta_{4}=\delta_{10}=\delta_{12}=1$ and $\delta_{8}=2$. If we set $r_{i}$ the remainder of the division of $u_{i} x_{4}^{\delta_{i}}$ for all $i \in\{4,8,10,12\}$, we get that

- $r_{4}=-x_{4} x_{5}^{2} b_{1}+2 x_{4} x_{5} b_{4}+x_{5} b_{6}+x_{5} b_{7}$, 
- $r_{8}=x_{4}^{2} x_{5} b_{3}+x_{5} b_{11}$,

- $r_{10}=x_{4}^{2} x_{5} b_{2}+3 x_{4} x_{5} b_{8}+\left(x_{5}^{2}-x_{4} x_{5}\right) b_{9}$, and

- $r_{12}=x_{4}^{2} x_{5}^{2} b_{1}+x_{4} x_{5} b_{6}+x_{5}^{2} b_{7}$.

Hence, we obtain the following minimal graded free resolution of $R / I$

$$
\mathcal{F}: 0 \rightarrow A(-3) \oplus A^{3}(-4) \stackrel{\psi}{\rightarrow} A \oplus A^{3}(-1) A^{5}(-2) \oplus A^{3}(-3) \rightarrow R / I \rightarrow 0,
$$

where $\psi$ is given by the matrix

$$
\left(\begin{array}{cccc}
x_{4} x_{5}^{2} & 0 & 0 & -x_{4}^{2} x_{5}^{2} \\
0 & 0 & -x_{4}^{2} x_{5} & 0 \\
0 & 0 & 0 & 0 \\
-2 x_{4} x_{5} & -x_{4}^{2} x_{5} & 0 & 0 \\
x_{4} & 0 & 0 & 0 \\
-x_{5} & 0 & 0 & -x_{4} x_{5} \\
-x_{5} & 0 & 0 & -x_{5}^{2} \\
0 & x_{4}^{2} & -3 x_{4} x_{5} & 0 \\
0 & 0 & x_{4} x_{5}-x_{5}^{2} & 0 \\
0 & 0 & x_{4} & 0 \\
0 & -x_{5} & 0 & 0 \\
0 & 0 & 0 & x_{4}
\end{array}\right)
$$

Moreover, the Hilbert series of $R / I$ is

$$
H S_{R / I}(t)=\frac{1+3 t+5 t^{2}+2 t^{3}-3 t^{4}}{(1-t)^{2}}
$$

and $\operatorname{reg}(R / I)=\max \{3,4-1\}=3$.

\section{Noether Resolution. Simplicial SEMigroup RingS}

This section concerns the study of Noether resolutions in simplicial semigroup rings $R / I$, i.e., whenever $I=I_{\mathcal{A}}$ with $\mathcal{A}=\left\{a_{1}, \ldots, a_{n}\right\} \subset \mathbb{N}^{d}$ and $a_{n-d+i}=w_{n-d+i} e_{i}$ for all $i \in\{1, \ldots, d\}$, where $\left\{e_{1}, \ldots, e_{d}\right\}$ is the canonical basis of $\mathbb{N}^{d}$. In this setting, $R / I_{\mathcal{A}}$ is isomorphic to the semigroup ring $K[\mathcal{S}]$, where $\mathcal{S}$ is the simplicial semigroup generated by $\mathcal{A}$. When $K$ is infinite, $I_{\mathcal{A}}$ is the vanishing ideal of the variety given parametrically by $x_{i}:=t^{a_{i}}$ for all $i \in\{1, \ldots, n\}$ (see, e.g., [Villarreal (2015)] ) and, hence, $K[\mathcal{S}]$ is the coordinate ring of a parametric variety. In this section we study the multigraded Noether resolution of $K[\mathcal{S}]$ with respect to the multigrading $\operatorname{deg}_{\mathcal{S}}\left(x_{i}\right)=a_{i} \in \mathcal{S}$; namely,

$$
\mathcal{F}: 0 \longrightarrow \oplus_{s \in \mathcal{S}_{p}} A \cdot s \stackrel{\psi_{p}}{\longrightarrow} \cdots \stackrel{\psi_{1}}{\longrightarrow} \oplus_{s \in \mathcal{S}_{0}} A \cdot s \stackrel{\psi_{0}}{\longrightarrow} K[\mathcal{S}] \longrightarrow 0 .
$$

where $\mathcal{S}_{i} \subset \mathcal{S}$ for all $i \in\{0, \ldots, p\}$. We observe that this multigrading is a refinement of the grading given by $\omega=\left(\omega_{1}, \ldots, \omega_{n}\right)$ with $\omega_{i}:=\sum_{j=1}^{d} a_{i j} \in \mathbb{Z}^{+}$; thus, $I_{\mathcal{A}}$ is $\omega$-homogeneous and the results of the previous section also apply here.

Our objective is to provide a description of this resolution in terms of the semigroup $\mathcal{S}$. We completely achieve this goal when $K[\mathcal{S}]$ is Cohen-Macaulay (which includes the case $d=1$ ) and also when $d=2$.

For any value of $d \geq 1$, the first step of the resolution corresponds to a minimal set of generators of $K[\mathcal{S}]$ as $A$-module and is given by the following well known result.

Proposition 5. Let $K[\mathcal{S}]$ be a simplicial semigroup ring. Then,

$$
\mathcal{S}_{0}=\left\{s \in \mathcal{S} \mid s-a_{i} \notin \mathcal{S} \text { for all } i \in\{n-d+1, \ldots, n\}\right\} .
$$

Moreover, $\psi_{0}: \oplus_{s \in \mathcal{S}_{0}} A \cdot s \longrightarrow K[\mathcal{S}]$ is the homomorphism of A-modules induced by $e_{s} \mapsto t^{s}$, where $\left\{e_{s} \mid s \in \mathcal{S}_{0}\right\}$ is the canonical basis of $\oplus_{s \in \mathcal{S}_{0}} A \cdot s$. 
Proposition 5 gives us the whole multigraded Noether resolution of $K[\mathcal{S}]$ when $K[\mathcal{S}]$ is CohenMacaulay.

In [Goto et al. (1976), Theorem 1] (see also [Stanley (1978), Theorem 6.4]), the authors provide a characterization of the Cohen-Macaulay property of $K[\mathcal{S}]$. In the following result we are proving an equivalent result that characterizes this property in terms of the size of $\mathcal{S}_{0}$. The proof shows how to obtain certain elements of $\operatorname{Ker}\left(\psi_{0}\right)$ and this idea will be later exploited to describe the whole resolution when $d=2$ and $K[\mathcal{S}]$ is not Cohen-Macaulay.

Proposition 6. Let $\mathcal{S}$ be a simplicial semigroup as above. Set $D:=\left(\prod_{i=1}^{d} \omega_{n-d+i}\right) /\left[\mathbb{Z}^{d}: \mathbb{Z} \mathcal{S}\right]$, where $\left[\mathbb{Z}^{d}: \mathbb{Z} \mathcal{S}\right]$ denotes the index of the group generated by $\mathcal{S}$ in $\mathbb{Z}^{d}$. Then, $K[\mathcal{S}]$ is CohenMacaulay $\Longleftrightarrow\left|\mathcal{S}_{0}\right|=D$.

Proof. By Auslander-Buchsbaum formula we deduce that $K[\mathcal{S}]$ is Cohen-Macaulay if and only if $\psi_{0}$ is injective, where $\psi_{0}$ is the morphism given in Proposition 5. We are proving that $\psi_{0}$ is injective if and only if $\left|\mathcal{S}_{0}\right|=D$. We define an equivalence relation on $\mathbb{Z}^{d}, u \sim v \Longleftrightarrow u-v \in$ $\mathbb{Z}\left\{\omega_{n-d+1} e_{1}, \ldots, \omega_{n} e_{d}\right\}$. This relation partitions $\mathbb{Z} \mathcal{S}$ into $D=\left[\mathbb{Z} \mathcal{S}: \mathbb{Z}\left\{\omega_{n-d+1} e_{1}, \ldots, \omega_{n} e_{d}\right\}\right]$ equivalence classes. Since

$$
\mathbb{Z}^{d} / \mathbb{Z} \mathcal{S} \simeq\left(\mathbb{Z}^{d} / \mathbb{Z}\left\{\omega_{n-d+1} e_{1}, \ldots, \omega_{n} e_{d}\right\}\right) /\left(\mathbb{Z} \mathcal{S} / \mathbb{Z}\left\{\omega_{n-d+1} e_{1}, \ldots, \omega_{n} e_{d}\right\}\right),
$$

we get that $D=\left(\prod_{i=1}^{d} \omega_{n-d+i}\right) /\left[\mathbb{Z}^{d}: \mathbb{Z} \mathcal{S}\right]$. Moreover, the following two facts are easy to check: for every equivalence class there exists an element $b \in \mathcal{S}_{0}$, and $\mathcal{S}=\mathcal{S}_{0}+\mathbb{N}\left\{\omega_{n-d+1} e_{1}, \ldots, \omega_{n} e_{d}\right\}$. This proves that $\left|\mathcal{S}_{0}\right| \geq D$.

Assume that $\left|\mathcal{S}_{0}\right|>D$, then there exist $u, v \in \mathcal{S}_{0}$ such that $u \sim v$ or, equivalently, $u+$ $\sum_{i=1}^{d} \lambda_{i} \omega_{n-d+i} e_{i}=v+\sum_{i=1}^{d} \mu_{i} \omega_{n-d+i} e_{i}$ for some $\lambda_{i}, \mu_{i} \in \mathbb{N}$ for all $i \in\{1, \ldots, d\}$. Thus $x_{n-d+1}^{\lambda_{1}} \cdots x_{n}^{\lambda_{d}} e_{u}-x_{n-d+1}^{\mu_{1}} \cdots x_{n}^{\mu_{d}} e_{v} \in \operatorname{Ker}\left(\psi_{0}\right)$ and $\psi_{0}$ is not injective.

Assume now that $\left|\mathcal{S}_{0}\right|=D$, then for every $s_{1}, s_{2} \in \mathcal{S}_{0}, s_{1} \neq s_{2}$, we have that $s_{1} \not s_{2}$. As a consequence, an element $\rho \in \oplus_{s \in \mathcal{S}_{0}} A \cdot s$ is homogeneous if and only if it is a monomial, i.e., $\rho=c x^{\alpha} e_{s}$ for some $c \in K, x^{\alpha} \in A$ and $s \in \mathcal{S}_{0}$. Since the image by $\psi_{0}$ of a monomial is another monomial, then there are no homogeneous elements in $\operatorname{Ker}\left(\psi_{0}\right)$ different from 0 , so $\psi_{0}$ is injective.

From now on suppose that $K[\mathcal{S}]$ is a 2-dimensional non Cohen-Macaulay semigroup ring. In this setting, we consider the set

$$
\Delta:=\left\{s \in \mathcal{S} \mid s-a_{n-1}, s-a_{n} \in \mathcal{S} \text { and } s-a_{n}-a_{n-1} \notin \mathcal{S}\right\} .
$$

The set $\Delta$ or slight variants of it has been considered by other authors (see, e.g., [Goto et al. (1976), Stanley (1978), Trung \& Hoa (1986)] ). We claim that $\Delta$ has exactly $\left|\mathcal{S}_{0}\right|-D$ elements. Indeed, if we consider the equivalence relation $\sim$ of Proposition 6, then $\sim$ partitions $\mathbb{Z} \mathcal{S}$ in $D$ classes $C_{1}, \ldots, C_{D}$ and it is straightforward to check that $\left|\Delta \cap C_{i}\right|=\left|\mathcal{S}_{0} \cap C_{i}\right|-1$ for all $i \in\{1, \ldots, D\}$. From here, we easily deduce that $|\Delta|=\left|\mathcal{S}_{0}\right|-D$. Hence, a direct consequence of Proposition 6 is that $\Delta$ is nonempty because $K[\mathcal{S}]$ is not Cohen-Macaulay. Furthermore, as Theorem 2 shows, the set $\Delta$ is not only useful to characterize the Cohen-Macaulay property but also provides the set of shifts in the second step of the multigraded Noether resolution of $K[\mathcal{S}]$.

Theorem 2. Let $K[\mathcal{S}]$ be a 2-dimensional semigroup ring and let

$$
\Delta=\left\{s \in \mathcal{S} \mid s-a_{n-1}, s-a_{n} \in \mathcal{S} \text { and } s-a_{n}-a_{n-1} \notin \mathcal{S}\right\},
$$

as above. Then, $\mathcal{S}_{1}=\Delta$.

Proof. Set $\mathcal{B}_{0}$ the monomial basis of $R /\left(\operatorname{in}\left(I_{\mathcal{A}}\right), x_{n-1}, x_{n}\right)$, where in $\left(I_{\mathcal{A}}\right)$ is the initial ideal of $I_{\mathcal{A}}$ with respect to $>_{\omega}$. For every $u=x_{1}^{\alpha_{1}} \cdots x_{n}^{\alpha_{n}} \in \mathcal{B}_{1}$ we set $\delta_{u} \geq 1$ the minimum integer such that $u x_{n-1}^{\delta_{u}} \in \operatorname{in}\left(I_{\mathcal{A}}\right)$. Consider $p_{u} \in R$ the remainder of $u x_{n-1}^{\delta_{u}}$ modulo the reduced Gröbner basis of 
$I_{\mathcal{A}}$ with respect to $>_{\omega}$, then $u x_{n-1}^{\delta_{u}}-p_{u} \in I_{\mathcal{A}}$. Since $I_{\mathcal{A}}$ is a binomial ideal, we get that $p_{u}=x^{\gamma}$ for some $\left(\gamma_{1}, \ldots, \gamma_{n}\right) \in \mathbb{N}^{n}$. Moreover, the condition $x^{\alpha}>x^{\gamma}$ and the minimality of $\delta_{u}$ imply that $\gamma_{n}>0$ and $\gamma_{n-1}=0$, so $x^{\gamma}=v_{u} x_{n}^{\gamma_{u}}$ with $v_{u} \in \mathcal{B}_{0}$. As we proved in Proposition 4, if we denote by $\left\{e_{v} \mid v \in \mathcal{B}_{0}\right\}$ the canonical basis of $\oplus_{v \in \mathcal{B}_{0}} A\left(-\operatorname{deg}_{\mathcal{S}}(v)\right)$ and $h_{u}:=x_{n-1}^{\delta_{u}} e_{u}-x_{n}^{\gamma_{v}} e_{v_{u}}$ for all $u \in \mathcal{B}_{1}$, then $\operatorname{Ker}\left(\psi_{0}\right)$ is the $A$-module minimally generated by $\mathcal{C}:=\left\{h_{u} \mid u \in \mathcal{B}_{1}\right\}$. Let us prove that

$$
\left\{\operatorname{deg}_{\mathcal{S}}\left(h_{u}\right) \mid u \in \mathcal{B}_{1}\right\}=\left\{s \in \mathcal{S} \mid s-a_{n-1}, s-a_{n} \in \mathcal{S} \text { and } s-a_{n-1}-a_{n} \notin \mathcal{S}\right\} .
$$

Take $s=\operatorname{deg}_{\mathcal{S}}\left(h_{u}\right)$ for some $u \in \mathcal{B}_{1}$, then $s=\operatorname{deg}_{\mathcal{S}}\left(h_{u}\right)=\operatorname{deg}_{\mathcal{S}}(u)+\delta_{u} a_{n-1}=\operatorname{deg}_{\mathcal{S}}\left(v_{u}\right)+\gamma_{v_{u}} a_{n}$. Since $\delta_{u}, \gamma_{v_{u}} \geq 1$, we get that both $s-a_{n-1}, s-a_{n} \in \mathcal{S}$. Moreover, if $s-a_{n-1}-a_{n}=\sum_{i=1}^{n} \delta_{i} a_{i} \in$ $\mathcal{S}$, then $x_{n-1}^{\delta_{u}-1} u-x^{\lambda} x_{n+1} \in I_{\mathcal{A}}$, which contradicts the minimality of $\delta_{u}$.

Take now $s \in \mathcal{S}$ such that $s-a_{n-1}, s-a_{n} \in \mathcal{S}$ and $s-a_{n-1}-a_{n} \notin \mathcal{S}$. Since $s-a_{n-1}, s-a_{n} \in \mathcal{S}$, there exists $s^{\prime}, s^{\prime \prime} \in \mathcal{S}_{0}$ and $\gamma_{1}, \gamma_{2}, \lambda_{1}, \lambda_{2} \in \mathbb{N}$ such that $s-a_{n}=s^{\prime}+\gamma_{1} a_{n-1}+\gamma_{2} a_{n}$ and $s-a_{n+1}=$ $s^{\prime \prime}+\lambda_{1} a_{n-1}+\lambda_{2} a_{n}$. Observe that $\gamma_{2}=0$, otherwise $s-a_{n-1}-a_{n}=s^{\prime}+\gamma_{1} a_{n-1}+\left(\gamma_{2}-1\right) a_{n} \in \mathcal{S}$, a contradiction. Analogously $\lambda_{1}=0$. Take $u, v \in \mathcal{B}_{0} \operatorname{such}$ that $\operatorname{deg}_{\mathcal{S}}(u)=s^{\prime}$ and $\operatorname{deg}_{\mathcal{S}}(v)=s^{\prime \prime}$. We claim that $u \in J$ and that $\delta_{u}=\gamma_{1}$. Indeed, $f:=u x_{n-1}^{\gamma_{1}}-v x_{n}^{\lambda_{2}} \in I_{\mathcal{A}}$ and in $(f)=u x_{n-1}^{\gamma_{1}}$, so $u \in \mathcal{B}_{1}$. Moreover, if there exists $\gamma^{\prime}<\delta_{u}$, then $s-a_{n-1}-a_{n} \in \mathcal{S}$, a contradiction.

One of the interests of Proposition 6 and Theorem 2 is that they describe multigraded Noether resolutions of dimension 2 semigroup rings in terms of the semigroup $\mathcal{S}$ and, in particular, they do not depend on the characteristic of the field $K$.

Now we consider the multigraded Hilbert Series of $K[\mathcal{S}]$, which is defined by

$$
H S_{K[\mathcal{S}]}(t)=\sum_{s \in \mathcal{S}} t^{s}=\sum_{s=\left(s_{1}, \ldots, s_{d}\right) \in \mathcal{S}} t_{1}^{s_{1}} \cdots t_{d}^{s_{d}}
$$

When $d=2$, from the description of the multigraded Noether resolution of $K[\mathcal{S}]$ we derive an expression of its multigraded Hilbert series in terms of $\mathcal{S}_{0}$ and $\mathcal{S}_{1}$.

Corollary 3. Let $K[\mathcal{S}]$ be a dimension 2 semigroup ring. The multigraded Hilbert series of $K[\mathcal{S}]$ is:

$$
H S_{K[\mathcal{S}]}(t)=\frac{\sum_{s \in \mathcal{S}_{0}} t^{s}-\sum_{s \in \mathcal{S}_{1}} t^{s}}{\left(1-t_{1}^{\omega_{n-1}}\right)\left(1-t_{2}^{\omega_{n}}\right)} .
$$

Remark 1. When $K[\mathcal{S}]$ is a two dimensional semigroup ring and $\mathcal{S}$ is generated by the set $\mathcal{A}=$ $\left\{a_{1}, \ldots, a_{n}\right\} \subset \mathbb{N}^{2}$, if we set $\omega=\left(\omega_{1}, \ldots, \omega_{n}\right) \in \mathbb{N}^{n}$ with $\omega_{i}:=a_{i, 1}+a_{i, 2}$ for all $i \in\{1, \ldots, n\}$, then $I_{\mathcal{A}}$ is $\omega$-homogeneous, as observed at the beginning of this section. The Noether resolution of $K[\mathcal{S}]$ with respect to this grading is easily obtained from the multigraded one. Indeed, it is given by the following expression:

$$
\mathcal{F}: 0 \longrightarrow \oplus_{\left(b_{1}, b_{2}\right) \in \mathcal{S}_{1}} A\left(-\left(b_{1}+b_{2}\right)\right) \stackrel{\psi_{1}}{\longrightarrow} \oplus_{\left(b_{1}, b_{2}\right) \in \mathcal{S}_{0}} A\left(-\left(b_{1}+b_{2}\right)\right) \stackrel{\psi_{0}}{\longrightarrow} K[\mathcal{S}] \longrightarrow 0 .
$$

In addition, the weighted Hilbert series of $K[\mathcal{S}]$ is obtained from the multigraded one by just considering the transformation $t_{1}^{\alpha_{1}} t_{2}^{\alpha_{2}} \mapsto t^{\alpha_{1}+\alpha_{2}}$.

When $\omega_{1}=\cdots=\omega_{n}$, then $I_{\mathcal{A}}$ is a homogeneous ideal. In this setting, the Noether resolution with respect to the standard grading is

$$
\mathcal{F}: 0 \longrightarrow \oplus_{\left(b_{1}, b_{2}\right) \in \mathcal{S}_{1}} A\left(-\left(b_{1}+b_{2}\right) / \omega_{1}\right) \stackrel{\psi_{1}}{\longrightarrow} \oplus_{\left(b_{1}, b_{2}\right) \in \mathcal{S}_{0}} A\left(-\left(b_{1}+b_{2}\right) / \omega_{1}\right) \stackrel{\psi_{0}}{\longrightarrow} K[\mathcal{S}] \longrightarrow 0 .
$$

Thus, the Castelnuovo-Mumford regularity of $K[\mathcal{S}]$ is

$$
\operatorname{reg}(K[\mathcal{S}])=\max \left(\left\{\frac{b_{1}+b_{2}}{\omega_{1}} \mid\left(b_{1}, b_{2}\right) \in \mathcal{S}_{0}\right\} \cup\left\{\frac{b_{1}+b_{2}}{\omega_{1}}-1 \mid\left(b_{1}, b_{2}\right) \in \mathcal{S}_{1}\right\}\right) .
$$

Moreover, the Hilbert series of $K[\mathcal{S}]$ is obtained from the multigraded Hilbert series by just considering the transformation $t_{1}^{\alpha_{1}} t_{2}^{\alpha_{2}} \mapsto t^{\left(\alpha_{1}+\alpha_{2}\right) / \omega_{1}}$. 


\section{MACAULAYFICATION OF SIMPLICIAL SEMIGROUP RINGS}

Given $K[\mathcal{S}]$ a simplicial semigroup ring, the semigroup ring $K\left[\mathcal{S}^{\prime}\right]$ is the Macaulayfication of $K[\mathcal{S}]$ if the three following conditions are satisfied:

(1) $\mathcal{S} \subset \mathcal{S}^{\prime}$,

(2) $K\left[\mathcal{S}^{\prime}\right]$ is Cohen-Macaulay, and

(3) the Krull dimension of $K\left[\mathcal{S}^{\prime} \backslash \mathcal{S}\right]$ is $\leq d-2$, where $d$ is the Krull dimension of $K[\mathcal{S}]$.

The existence and uniqueness of a $K\left[\mathcal{S}^{\prime}\right]$ fulfilling the previous properties for simplicial semigroup rings is guaranteed by [Morales (2007), Theorem 5]. In this section we describe explicitly the Macaulayfication of any simplicial semigroup ring in terms of the set $\mathcal{S}_{0}$. For this purpose we consider the same equivalence relation in $\mathbb{Z}^{d}$ as in proof of Proposition 6 , namely, for $s_{1}, s_{2} \in \mathbb{Z}^{d}$

$$
s_{1} \sim s_{2} \Longleftrightarrow s_{1}-s_{2} \in \mathbb{Z}\left\{\omega_{n-d+1} e_{1}, \ldots, \omega_{n} e_{d}\right\} .
$$

As we have seen, $\mathcal{S}_{0} \subset \mathbb{Z}^{d}$ is partitioned into $D:=\omega_{n-d+1} \cdots \omega_{n} /\left[\mathbb{Z}^{d}: \mathbb{Z} \mathcal{S}\right]$ equivalence classes $S^{1}, \ldots, S^{D}$. For every equivalence class $S^{i}$ we define a vector $b_{i}$ in the following way. We take $S^{i}=\left\{s_{1}, \ldots, s_{t}\right\}$, where $s_{j}=\left(s_{j 1}, \ldots, s_{j d}\right) \in \mathbb{N}^{d}$ for all $j \in\{1, \ldots, t\}$ and define $b_{i}=\left(b_{i 1}, \ldots, b_{i d}\right) \in \mathbb{N}^{d}$ as the vector whose $k$-th coordinate $b_{i k}$ equals the minimum of the $k$ th coordinates of $s_{1}, \ldots, s_{t}$, this is, $b_{i k}:=\min \left\{s_{1 k}, \ldots, s_{t k}\right\}$. We denote $\mathfrak{B}:=\left\{b_{1}, \ldots, b_{D}\right\}$ and

$$
\mathcal{S}^{\prime}:=\mathfrak{B}+\mathbb{N}\left\{\omega_{n-d+1} e_{1}, \ldots, \omega_{n} e_{d}\right\} .
$$

The objective of this section is to prove that $K\left[\mathcal{S}^{\prime}\right]$ is the Macaulayfication of $K[\mathcal{S}]$. The main issue in the proof is to show that $\operatorname{dim}\left(K\left[\mathcal{S}^{\prime} \backslash \mathcal{S}\right]\right) \leq d-2$. For this purpose we use a technique developed in [Morales \& Nhan (2003)] which consists of determining the dimension of a graded ring by studying its Hilbert function. More precisely, for $L$ an $\omega$-homogeneous ideal, if we denote by $h(i)$ the Hilbert function of $R / L$, by [Morales (1985), Lemma 1.4], there exist some polynomials $Q_{1}, \ldots, Q_{s} \in \mathbb{Z}[t]$ with $s \in \mathbb{Z}^{+}$such that $h(l s+i)=Q_{i}(l)$ for all $i \in\{1, \ldots, s\}$ and $l \in \mathbb{Z}^{+}$ large enough. Moreover, in [Morales (2016)], the author proves the following.

Theorem 3. Let $L$ be a $\omega$-homogeneous ideal and denote by $h: \mathbb{N} \rightarrow \mathbb{N}$ the Hilbert function of $R / L$. If we set $h^{0}(n)=\sum_{i=0}^{n} h(i)$, then there exist s polynomials $f_{1}, \ldots, f_{s} \in \mathbb{Z}[t]$ such that $h^{0}(l s+i)=f_{i}(l)$ for all $i \in\{1, \ldots, s\}$ and $l \in \mathbb{Z}^{+}$large enough. Moreover, all these polynomials $f_{1}, \ldots, f_{s}$ have the same leading term $c t^{\operatorname{dim}(R / L)} /(\operatorname{dim}(R / L))$ ! with $c \in \mathbb{Z}^{+}$.

In the proof of Theorem 4, we relate the Hilbert function of $K\left[\mathcal{S}^{\prime} \backslash \mathcal{S}\right]$ with that of several monomial ideals and use of the following technical lemma.

Lemma 1. Let $M \subset K\left[y_{1}, \ldots, y_{d}\right]$ be a monomial ideal. If for all $i \in\{1, \ldots, d\}$ there exist $x^{\alpha} \in M$ such that $x_{i} \nmid x^{\alpha}$, then $\operatorname{dim}\left(K\left[y_{1}, \ldots, y_{d}\right] / M\right) \leq d-2$.

Proof. Let us prove that $M$ has height $\geq 2$. By contradiction, assume that $M$ has an associated prime $\mathfrak{P}$ of height one. Since $M$ is monomial, then so is $\mathfrak{P}$. Therefore, $\mathfrak{P}=\left(x_{i}\right)$ for some $i \in\{1 \ldots, d\}$. Hence we get that $M \subset \sqrt{M} \subset \mathfrak{P}=\left(x_{i}\right)$, a contradiction.

Now we can proceed with the proof of the main result of this section.

Theorem 4. Let $K[\mathcal{S}]$ be a simplicial semigroup ring and let $\mathcal{S}^{\prime}$ be the semigroup described in (3). Then, $K\left[\mathcal{S}^{\prime}\right]$ is the Macaulayfication of $K[\mathcal{S}]$.

Proof. Is is clear that $\mathcal{S} \subset \mathcal{S}^{\prime}$. In order to obtain the result it suffices to prove that $\mathcal{S}^{\prime}$ is a semigroup, that $K\left[\mathcal{S}^{\prime}\right]$ is Cohen-Macaulay and that $\operatorname{dim}\left(K\left[\mathcal{S}^{\prime} \backslash \mathcal{S}\right]\right) \leq \operatorname{dim}(K[\mathcal{S}])-2$ (see, e.g., [Morales (2007)]).

Let us first prove that $\mathcal{S}^{\prime}$ is a semigroup. Take $s_{1}, s_{2} \in \mathcal{S}^{\prime}$, then there exists $i, j \in\{1, \ldots, D\}$ such that $s_{1}=b_{i}+c_{1}$ and $s_{2}=b_{j}+c_{2}$ for some $c_{1}, c_{2} \in \mathbb{N}\left\{\omega_{n-d+1} e_{1}, \ldots, \omega_{n} e_{d}\right\}$. Then $s_{1}+s_{2}=$ $b_{i}+b_{j}+c_{1}+c_{2}$. We take $k \in\{1, \ldots, D\}$ such that $b_{k} \sim b_{i}+b_{j}$. By construction of $\mathfrak{B}$ we have that $b_{k}=b_{i}+b_{j}+c_{3}$ for some $c_{3} \in\left\{\omega_{n-d+1} e_{1}, \ldots, \omega_{n} e_{d}\right\}$ and, hence, $s_{1}+s_{2} \in \mathcal{S}^{\prime}$. 
To prove that $\mathcal{S}^{\prime}$ is Cohen-Macaulay it suffices to observe that $\mathfrak{B}=\left\{b \in \mathcal{S}^{\prime} \mid b-a_{i} \notin \mathcal{S}^{\prime}\right.$ for all $i \in\{1, \ldots, d\}\}$ and that $|\mathfrak{B}|=D$, so by Proposition 6 it follows that $\mathcal{S}^{\prime}$ is Cohen-Macaulay.

Let us prove that $\operatorname{dim}\left(K\left[\mathcal{S}^{\prime} \backslash \mathcal{S}\right]\right) \leq d-2$. For all $s=\left(s_{1}, \ldots, s_{m}\right) \in \mathbb{N}^{m}$ we consider the grading $\operatorname{deg}\left(t^{s}\right)=\sum_{i=1}^{m} s_{i}$ and we denote $h, h^{\prime}$ and $\widehat{h}$ the Hilbert functions of $K[\mathcal{S}], K\left[\mathcal{S}^{\prime}\right]$ and $K\left[\mathcal{S}^{\prime} \backslash\right.$ $\mathcal{S}]$ respectively, then $\widehat{h}=h^{\prime}-h$. Moreover, we have that $h^{\prime}=\sum_{i=1}^{D} h_{i}^{\prime}$ and $h=\sum_{i=1}^{D} h_{i}$ where $h_{i}^{\prime}(d):=\mid\left\{s \in \mathcal{S}^{\prime} \mid \operatorname{deg} t^{s}=d\right.$ and $\left.s \sim b_{i}\right\} \mid$ and $h_{i}(d):=\mid\left\{s \in \mathcal{S} \mid \operatorname{deg} t^{s}=d\right.$ and $\left.s \sim b_{i}\right\} \mid$. For each $i \in\{1, \ldots, D\}$ we define a monomial ideal $M_{i} \subset k\left[y_{1}, \ldots, y_{d}\right]$ as follows: for every $b \in \mathcal{S}$ such that $b \sim b_{i}$ we define the monomial $m_{b}:=y_{1}^{\beta_{1}} \cdots y_{d}^{\beta_{d}}$ if $b=b_{i}+\sum_{i=1}^{d} \beta_{i} \omega_{n-d+i} e_{i}$ and $M_{i}:=\left(\left\{m_{b} \mid b \in \mathcal{S}, b \sim b_{i}\right\}\right)$. We consider in $K\left[y_{1}, \ldots, y_{d}\right]$ the grading $\operatorname{deg}_{\omega}\left(y_{i}\right)=\omega_{n-d+i}$ and denote by $h_{i}^{\omega}$ the corresponding $\omega$-homogeneous Hilbert function of $K\left[y_{1}, \ldots, y_{d}\right] / M_{i}$. We have the following equality $h_{i}^{\omega}(\lambda)=h_{i}^{\prime}\left(\sum_{j=1}^{d} b_{i j}+\lambda\right)-h_{i}\left(\sum_{j=1}^{d} b_{i_{j}}+\lambda\right)$ because $y^{\beta} \notin M_{i} \Longleftrightarrow$ $b_{i}+\sum_{i=1}^{d} \beta_{i} \omega_{n-d+i} e_{i} \in \mathcal{S}^{\prime} \backslash \mathcal{S}$. Hence, we have expressed the Hilbert function $\widehat{h}$ of $K\left[\mathcal{S} \backslash \mathcal{S}^{\prime}\right]$ as a sum of $D$ Hilbert functions of $K\left[y_{1}, \ldots, y_{d}\right] / M_{i}$, for some monomial ideals $M_{1}, \ldots, M_{D}$ and, by Lemma 1, $\operatorname{dim}\left(K\left[y_{1}, \ldots, y_{d}\right] / M_{i}\right) \leq d-2$. Thus, by Theorem 3 , we can conclude that the dimension of $K\left[\mathcal{S}^{\prime} \backslash \mathcal{S}\right]$ equals the maximum of $\operatorname{dim}\left(K\left[y_{1}, \ldots, y_{d}\right] / M_{i}\right) \leq d-2$ and we get the result.

We finish this section with an example showing how to compute the Macaulayfication by means of the set $\mathcal{S}_{0}$. Moreover, this example illustrates that even if $K[\mathcal{S}]=R / I_{\mathcal{A}}$ with $I_{\mathcal{A}}$ a homogeneous ideal, it might happen that the ideal associated to $K\left[\mathcal{S}^{\prime}\right]$ is not standard homogeneous.

Example 3. We consider the semigroup ring $K[\mathcal{S}]$, where $\mathcal{S} \subset \mathbb{N}^{2}$ is the semigroup generated by $\mathcal{A}:=\{(1,9),(4,6),(5,5),(10,0),(0,10)\} \subset \mathbb{N}^{2}$. Then, $K[\mathcal{S}]=R / I_{\mathcal{A}}$ and $I_{\mathcal{A}}$ is homogeneous. If we compute the set $\mathcal{S}_{0}$ we get that

$$
\mathcal{S}_{0}=\{(0,0),(1,9),(2,18),(3,27),(13,17),(4,6),(5,5),(6,14),(7,23),(8,12),(9,11)\} .
$$

Moreover we compute $D=100 /\left[\mathbb{Z}^{d}: \mathbb{Z S}\right]=10$ and get $S^{1}=\{(0,0)\}, S^{2}=\{(1,9)\}$, $S^{3}=\{(2,18)\}, S^{4}=\{(3,27),(13,17)\}, S^{5}=\{(4,6)\}, S^{6}=\{(5,5)\}, S^{7}=\{(6,14)\}$, $S^{8}=\{(7,23)\}, S^{9}=\{(8,12)\}$ and $S^{10}=\{(9,11)\}$. So, the Macaulayfication $K\left[\mathcal{S}^{\prime}\right]$ of $K[\mathcal{S}]$ is given by $\mathcal{S}^{\prime}=\mathfrak{B}+\mathbb{N}\{(10,0),(0,10)\}$, where

$$
\mathfrak{B}=\{(0,0),(1,9),(2,18),(3,17),(4,6),(5,5),(6,14),(7,23),(8,12),(9,11)\} .
$$

Or equivalently, $\mathcal{S}^{\prime}$ is the semigroup generated by

$$
\mathcal{A}^{\prime}=\{(1,9),(3,17),(4,6),(5,5),(10,0),(0,10)\} .
$$

We observe that $K\left[\mathcal{S}^{\prime}\right] \simeq K\left[x_{1}, \ldots, x_{6}\right] / I_{\mathcal{A}^{\prime}}$ and that $I_{\mathcal{A}^{\prime}}$ is $\omega$-homogeneous with respect to $\omega=$ $(1,2,1,1,1,1)$ but not standard homogeneous.

\section{AN UPPER BOUND FOR THE CASTELNUOVO-MUMFORD REGULARITY OF PROJECTIVE MONOMIAL CURVES}

Every sequence $m_{1}<\ldots<m_{n}$ of relatively prime positive integers with $n \geq 2$ has associated the projective monomial curve $\mathcal{C} \subset \mathbb{P}_{K}^{n}$ given parametrically by $x_{i}:=s^{m_{i}} t^{m_{n}-m_{i}}$ for all $i \in$ $\{1, \ldots, n-1\}, x_{n}=s^{m_{n}}, x_{n+1}:=t^{m_{n}}$. If we set $\mathcal{A}:=\left\{a_{1}, \ldots, a_{n+1}\right\} \subset \mathbb{N}^{2}$ where $a_{i}:=$ $\left(m_{i}, m_{n}-m_{i}\right), a_{n}:=\left(m_{n}, 0\right)$ and $a_{n+1}:=\left(0, m_{n}\right)$, it turns out that $I_{\mathcal{A}} \subset K\left[x_{1}, \ldots, x_{n+1}\right]$ is the defining ideal of $\mathcal{C}$. If we denote by $\mathcal{S}$ the semigroup generated by $\mathcal{A}$, then the 2-dimensional semigroup ring $K[\mathcal{S}]$ is isomorphic to $K\left[x_{1}, \ldots, x_{n+1}\right] / I_{\mathcal{A}}$, the homogeneous coordinate ring of $\mathcal{C}$. Hence, the methods of the previous sections apply here to describe its multigraded Noether resolution, and the formula (2) in Remark 1 for the Castelnuovo-Mumford regularity holds in this context (with $\omega_{1}=m_{n}$ ). The goal of this section is to use this formula to prove Theorem 5 , which provides an upper bound for the Castelnuovo-Mumford regularity of $K[\mathcal{S}]$. The proof we are presenting is elementary and uses some classical results on numerical semigroups. We will 
introduce now the results on numerical semigroups that we need for our proof (for more on this topic we refer to [Rosales \& García-Sánchez (2009)] and [Ramírez Alfonsín (2005)]).

Given $m_{1}, \ldots, m_{n}$ a set of relatively prime integers, we denote by $\mathcal{R}$ the numerical subsemigroup of $\mathbb{N}$ spanned by $m_{1}, \ldots, m_{n}$. The largest integer that does not belong to $\mathcal{R}$ is called the Frobenius number of $\mathcal{R}$ and will be denoted by $\mathrm{g}(\mathcal{R})$. We consider the Apery set of $\mathcal{R}$ with respect to $m_{n}$, i.e., the set

$$
\operatorname{Ap}\left(\mathcal{R}, m_{n}\right):=\left\{a \in \mathcal{R} \mid a-m_{n} \notin \mathcal{R}\right\} .
$$

It is a well known and easy to check that $\operatorname{Ap}\left(\mathcal{R}, m_{n}\right)$ constitutes a full set of residues modulo $m_{n}$ (and, in particular, has $m_{n}$ elements) and that $\max \left(\operatorname{Ap}\left(\mathcal{R}, m_{n}\right)\right)=\mathrm{g}(\mathcal{R})+m_{n}$. We will also use an upper bound on $\mathrm{g}(\mathcal{R})$ which is a slight variant of the one given in [Selmer (1977)] (which was deduced from a result of [Erdös \& Graham (1972)] ]). The reason why we do not use Selmer's bound itself is that it is only valid under the additional hypothesis that $n \leq m_{1}$. This is not a restrictive hypothesis when studying numerical semigroups, because whenever $m_{1}<\cdots<m_{n}$ is a minimal set of generators of $\mathcal{R}$, then $n \leq m_{1}$. In our current setting of projective monomial curves, the case where $m_{1}<\cdots<m_{n}$ is not a minimal set of generators of $\mathcal{R}$ is interesting by itself (even the case $m_{1}=1$ is interesting); hence, a direct adaptation of the proof of Selmer yields that

$$
g(\mathcal{R}) \leq 2 m_{n}\left\lfloor\frac{m_{\tau}}{n}\right\rfloor-m_{\tau},
$$

for every $m_{\tau} \geq n$. Note that $m_{n} \geq n$ and then, such a value $\tau$ always exists..

We first include a result providing an upper bound for $\operatorname{reg}(K[\mathcal{S}])$ when $K[\mathcal{S}]$ is Cohen-Macaulay.

Proposition 7. Let $m_{1}<\ldots<m_{n}$ be a sequence of relatively prime positive integers with $n \geq 2$ and let $\tau \in\{1, \ldots, n\}$ such that $m_{\tau} \geq n$. If $K[\mathcal{S}]$ is Cohen-Macaulay, then

$$
\operatorname{reg}(K[\mathcal{S}]) \leq\left\lfloor\left(2 m_{n}\left\lfloor\frac{m_{\tau}}{n}\right\rfloor-m_{\tau}+m_{n}\right) / m_{1}\right\rfloor .
$$

In particular, if $m_{1} \geq n$, we have that $\operatorname{reg}(K[\mathcal{S}]) \leq\left\lfloor m_{n}\left(\frac{2}{n}+\frac{1}{m_{1}}\right)-1\right\rfloor$.

Proof. We consider the equivalence relation $\sim$ of Section 4 . Indeed, since now $\mathbb{Z} \mathcal{S}=\{(x, y) \mid x+$ $\left.y \equiv 0\left(\bmod m_{n}\right)\right\}$, then we have that $\sim$ partitions the set $\mathcal{S}_{0}$ in exactly $m_{n}$ equivalence classes. Moreover, since $K[\mathcal{S}]$ is Cohen-Macaulay, we have that

- each of these classes has a unique element,

- $\mathcal{S}_{1}=\emptyset$, and

- $\operatorname{reg}(K[\mathcal{S}])=\max \left\{\frac{b_{1}+b_{2}}{m_{n}} \mid\left(b_{1}, b_{2}\right) \in \mathcal{S}_{0}\right\}($ see Remark 1).

Let us take $\left(b_{1}, b_{2}\right) \in \mathcal{S}_{0}$, then $\left(b_{1}, b_{2}\right)=\sum_{i=1}^{n-1} \alpha_{i} a_{i}$ and $\left(b_{1}+b_{2}\right) / m_{n}=\sum_{i=1}^{n-1} \alpha_{i}$. Moreover, we claim that $b_{1} \in \operatorname{Ap}\left(\mathcal{R}, m_{n}\right)$. Otherwise, $b_{1}-m_{n} \in \mathcal{R}$ and there would be another element $\left(c_{1}, c_{2}\right) \in \mathcal{S}_{0}$ such that $\left(c_{1}, c_{2}\right) \sim\left(b_{1}, b_{2}\right)$, a contradiction. Hence, by (4),

$$
\left(\sum_{i=1}^{n-1} \alpha_{i}\right) m_{1} \leq \sum_{i=1}^{n-1} \alpha_{i} m_{i}=b_{1} \leq \mathrm{g}(\mathcal{R})+m_{n} \leq 2 m_{n}\left\lfloor\frac{m_{\tau}}{n}\right\rfloor-m_{\tau}+m_{n} .
$$

And, from this expression we conclude that

$$
\frac{b_{1}+b_{2}}{m_{n}}=\sum_{i=1}^{n-1} \alpha_{i} \leq\left(2 m_{n}\left\lfloor\frac{m_{\tau}}{n}\right\rfloor-m_{\tau}+m_{n}\right) / m_{1} .
$$

When $m_{1} \geq n$, then it suffices to take $\tau=1$ to get the result.

And now, we can prove the main result of the section. 
Theorem 5. Let $m_{1}<\ldots<m_{n}$ be a sequence of relatively prime positive integers with $n \geq 2$. If we take $\tau, \lambda$ such that $m_{\tau} \geq n$ and $m_{n}-m_{\lambda} \geq n$. Then,

$$
\operatorname{reg}(K[\mathcal{S}]) \leq\left\lfloor\frac{\left(2 m_{n}\left\lfloor\frac{m_{\tau}}{n}\right\rfloor-m_{\tau}+m_{n}\right)}{m_{1}}+\frac{\left(2 m_{n}\left\lfloor\frac{m_{n}-m_{\lambda}}{n}\right\rfloor+m_{\lambda}\right)}{\left(m_{n}-m_{n-1}\right)}\right\rfloor-2 .
$$

In particular, if $m_{1} \geq n$ and $m_{n}-m_{n-1} \geq n$, then $\operatorname{reg}(K[\mathcal{S}]) \leq\left\lfloor m_{n}\left(\frac{4}{n}+\frac{1}{m_{1}}+\frac{1}{m_{n}-m_{n-1}}\right)\right\rfloor-4$.

Proof. We consider $E$ one of the equivalence classes of $\mathbb{Z S}$ induced by the equivalence relation $\sim$ First, assume that $\mathcal{S}_{0} \cap E$ has a unique element which we call $\left(b_{1}, b_{2}\right)$. Then, $\mathcal{S}_{1} \cap E=\emptyset$, and the same argument as in the proof of Proposition 7 proves that $\frac{b_{1}+b_{2}}{m_{n}} \leq\left(2 m_{n}\left\lfloor\frac{m_{\tau}}{n}\right\rfloor-m_{\tau}+m_{n}\right) / m_{1}$.

Assume now that $\mathcal{S}_{0} \cap E=\left\{\left(x_{1}, y_{1}\right), \ldots,\left(x_{r}, y_{r}\right)\right\}$ with $r \geq 2$ and $x_{1}<x_{2}<\cdots<x_{r}$. We claim that the following properties hold:

(a) $x_{1} \equiv x_{2} \equiv \cdots \equiv x_{r}\left(\bmod m_{n}\right)$,

(b) $y_{1}>\cdots>y_{r}$ and $y_{1} \equiv y_{2} \equiv \cdots \equiv y_{r}\left(\bmod m_{n}\right)$,

(c) $x_{1} \in \operatorname{Ap}\left(\mathcal{R}, m_{n}\right)$,

(d) $y_{r} \in \operatorname{Ap}\left(\mathcal{R}^{\prime}, m_{n}\right)$, where $\mathcal{R}^{\prime}$ is the numerical semigroup generated by $m_{n}-m_{n-1}<$ $m_{n}-m_{n-2}<\cdots<m_{n}-m_{1}<m_{n}$

(e) $\mathcal{S}_{1} \cap E=\left\{\left(x_{2}, y_{1}\right),\left(x_{3}, y_{2}\right), \ldots,\left(x_{r}, y_{r-1}\right)\right\}$, and

(f) $\max \left\{\frac{b_{1}+b_{2}}{m_{n}} \mid\left(b_{1}, b_{2}\right) \in \mathcal{S}_{0} \cap E\right\} \leq \max \left\{\frac{b_{1}+b_{2}}{m_{n}} \mid\left(b_{1}, b_{2}\right) \in \mathcal{S}_{1} \cap E\right\}-1$.

Properties (a) and (b) are evident. To prove (c) and (d) it suffices to take into account the following facts: $\mathcal{S} \subset \mathcal{R} \times \mathcal{R}^{\prime}$, and for every $b_{1} \in \mathcal{R}, b_{2} \in \mathcal{R}^{\prime}$ there exist $c_{1}, c_{2} \in \mathbb{N}$ such that $\left(b_{1}, c_{2}\right),\left(c_{1}, b_{2}\right) \in$ $\mathcal{S}$. To prove (e) we first observe that

$$
\mathcal{S} \cap E=\left\{b+\lambda\left(m_{n}, 0\right)+\mu\left(0, m_{n}\right) \mid b \in \mathcal{S}_{0} \cap E, \lambda, \mu \in \mathbb{N}\right\} .
$$

Take now $(x, y) \in \mathcal{S}_{1} \cap E$ and we take the minimum value $i \in\{1, \ldots, r\}$ such that $(x, y)=$ $\left(x_{i}, y_{i}\right)+\lambda\left(m_{n}, 0\right)+\mu\left(0, m_{n}\right)$ with $\lambda, \mu \in \mathbb{N}$; we observe that

- $\lambda>0$; otherwise $(x, y)-\left(m_{n}, 0\right) \notin \mathcal{S}$,

- $\mu=0$; otherwise $(x, y)-\left(m_{n}, m_{n}\right)=\left(x_{i}, y_{i}\right)+(\lambda-1)\left(m_{n}, 0\right)+(\mu-1)\left(0, m_{n}\right) \in \mathcal{S}$, a contradiction,

- $y \geq y_{r-1}$; otherwise $i=r$ and, since $(x, y)-\left(0, m_{n}\right) \in \mathcal{S} \cap E$, we get that $\mu \geq 1$,

- $x \leq x_{i+1}$; otherwise $(x, y)=\left(x_{i+1}, y_{i+1}\right)+\lambda^{\prime}\left(m_{n}, 0\right)+\mu^{\prime}\left(0, m_{n}\right)$ with $\lambda^{\prime}, \mu^{\prime} \geq 1$, a contradiction, and

- $x \geq x_{i+1}$; otherwise $(x, y)-\left(0, m_{n}\right) \notin \mathcal{S}$.

Hence, $(x, y)=\left(x_{i+1}, y_{i}\right)$ and $\mathcal{S}_{1} \cap E \subseteq\left\{\left(x_{2}, y_{1}\right),\left(x_{3}, y_{2}\right), \ldots,\left(x_{r}, y_{r-1}\right)\right\}$. Take now $i \in$ $\{1, \ldots, r-1\}$, and consider $\left(x_{i+1}, y_{i}\right) \in \mathcal{S}$. Since $\left(x_{i}, y_{i}\right),\left(x_{i+1}, y_{i+1}\right) \in E, x_{i} \equiv x_{i+1}\left(\bmod m_{n}\right)$ and $y_{i} \equiv y_{i+1}\left(\bmod m_{n}\right)$, then $\left(x_{i+1}, y_{i}\right) \in E$. We also have that there exist $\gamma, \delta \in \mathbb{N}$ such that $\left(x_{i+1}, y_{i}\right)-\left(m_{n}, 0\right)=\left(x_{i}, y_{i}\right)+\gamma\left(m_{n}, 0\right) \in \mathcal{S}$ and $\left(x_{i+1}, y_{i}\right)-\left(0, m_{n}\right)=\left(x_{i+1}, y_{i+1}\right)+\delta\left(0, m_{n}\right) \in$ $\mathcal{S}$. We claim that $\left(x_{i+1}, y_{i}\right)-\left(m_{n}, m_{n}\right) \notin \mathcal{S}$. Otherwise there exists $j \in\{1, \ldots, r\}$ such that $\left(x_{i+1}-m_{n}, y_{i}-m_{n}\right)=\left(x_{j}, y_{j}\right)+\lambda^{\prime}\left(m_{n}, 0\right)+\mu^{\prime}\left(0, m_{n}\right)$; this is not possible since $x_{i+1}-m_{n}<x_{i+1}$ implies that $j \leq i$, and $y_{i}-m_{n}<y_{i}$ implies that $j \geq i+1$. Thus, $\left(x_{i+1}, y_{i}\right) \in \mathcal{S}_{1}$ and (e) is proved. Property (f) follows from (e).

Moreover, since $x_{1} \in \operatorname{Ap}\left(\mathcal{R}, m_{n}\right)$, the same argument as in Proposition 7 proves that

$$
\frac{x_{1}+y_{1}}{m_{n}} \leq\left(2 m_{n}\left\lfloor\frac{m_{\tau}}{n}\right\rfloor-m_{\tau}+m_{n}\right) / m_{1},
$$

and a similar argument with $y_{r} \in \operatorname{Ap}\left(\mathcal{R}^{\prime}, m_{n}\right)$ proves that

$$
\frac{x_{r}+y_{r}}{m_{n}} \leq\left(2 m_{n}\left\lfloor\frac{m_{n}-m_{\lambda}}{n}\right\rfloor+m_{\lambda}\right) /\left(m_{n}-m_{n-1}\right) .
$$


And, since,

$$
\frac{x_{i+1}+y_{i}}{m_{n}}-1 \leq \frac{x_{r}+y_{1}}{m_{n}}-1 \leq \frac{x_{1}+y_{1}}{m_{n}}+\frac{x_{r}+y_{r}}{m_{n}}-2,
$$

putting together (5), (6) and (7) we get the result. If $m_{1} \geq n$ and $m_{n}-m_{n-1} \geq n$, it suffices to take $\tau=1$ and $\lambda=n-1$ to prove the result.

It is not difficult to build examples such that the bound provided by Theorem 5 outperforms the bound of L'vovsky's. Let us see an example.

Example 4. Set $n \geq 6$ and consider $m_{i}=n+i$ for all $i \in\{1, \ldots, n-1\}$ and $m_{n}=3 n$, then we can take $\tau=1$ and $\lambda=n-1$ and apply Theorem 5 to prove that

$$
\operatorname{reg}(K[\mathcal{S}]) \leq\left\lfloor 3 n\left(\frac{4}{n}+\frac{1}{n+1}+\frac{1}{n+1}\right)\right\rfloor-4=13,
$$

meanwhile the result of L'vovsky provides an upper bound of $2 n+1$.

\section{Noether RESOlution AND MACAUlayficATION OF PROJECTIVE MONOMIAL CURVES ASSOCIATED TO ARITHMETIC SEQUENCES AND THEIR CANONICAL PROJECTIONS.}

Every sequence $m_{1}<\ldots<m_{n}$ of positive integers with $n \geq 2$ has associated the projective monomial curve $\mathcal{C} \subset \mathbb{P}_{K}^{n}$ given parametrically by $x_{i}:=s^{m_{i}} t^{m_{n}-m_{i}}$ for all $i \in\{1, \ldots, n-1\}, x_{n}=$ $s^{m_{n}}, x_{n+1}:=t^{m_{n}}$. If we set $\mathcal{A}:=\left\{a_{1}, \ldots, a_{n+1}\right\} \subset \mathbb{N}^{2}$ where $a_{i}:=\left(m_{i}, m_{n}-m_{i}\right), a_{n}:=\left(m_{n}, 0\right)$ and $a_{n+1}:=\left(0, m_{n}\right)$, it turns out that $I_{\mathcal{A}} \subset K\left[x_{1}, \ldots, x_{n+1}\right]$ is the defining ideal of $\mathcal{C}$. Moreover, if we denote by $\mathcal{S}$ the semigroup generated by $\mathcal{A}$, then $K[\mathcal{S}] \simeq K\left[x_{1}, \ldots, x_{n+1}\right] / I_{\mathcal{A}}$ is a dimension 2 semigroup ring and the methods of the previous sections apply here to describe its multigraded Noether resolution.

In [Li et. al (2012)], the authors studied the set $\mathcal{S}_{0}$ whenever $m_{1}<\cdots<m_{n}$ is an arithmetic sequence of relatively prime integers, i.e., there exist $d, m_{1} \in \mathbb{Z}^{+}$such that $m_{i}=m_{1}+(i-1) d$ for all $i \in\{1, \ldots, n\}$ and $\operatorname{gcd}\left\{m_{1}, d\right\}=1$. In particular, they obtained the following result.

Theorem 6. [Li et. al (2012), Theorem 3.4]

$$
\mathcal{S}_{0}=\left\{\left(\left\lceil\frac{j}{n-1}\right\rceil m_{n}-j d, j d\right) \mid j \in\left\{0, \ldots, m_{n}-1\right\}\right\}
$$

From the previous result and Proposition 6 we deduce that $K[\mathcal{S}]$ is Cohen-Macaulay (see also [Bermejo et al. (2017), Corollary 2.3]), we obtain the shifts of the only step of the multigraded Noether resolution and, by Corollary 2 , we also derive that $\operatorname{reg}(K[\mathcal{S}])=\left\lceil\left(m_{n}-1\right) /(n-1)\right\rceil$ (see also [Bermejo et al. (2017), Theorem 2.7]). In the rest of this section we are using the tools developed in the previous sections to study the canonical projections of $\mathcal{C}$, i.e., for all $r \in\{1, \ldots, n-1\}$ and $n \geq 3$ we aim at studying the curve $\mathcal{C}_{r}:=\pi_{r}(\mathcal{C})$ obtained as the image of $\mathcal{C}$ under the projection $\pi_{r}$ from $\mathbb{P}_{K}^{n}$ to $\mathbb{P}_{K}^{n-1}$ defined by $\left(p_{1}: \cdots: p_{n+1}\right) \mapsto\left(p_{1}: \cdots: p_{r-1}: p_{r+1}: \cdots: p_{n+1}\right)$. We know that the vanishing ideal of $\mathcal{C}_{r}$ is $I_{\mathcal{A}_{r}}$, where $\mathcal{A}_{r}=\mathcal{A} \backslash\left\{a_{r}\right\}$ for all $r \in\{1, \ldots, n-1\}$. Note that $\mathcal{C}_{1}$ is the projective monomial curve associated to the arithmetic sequence $m_{2}<\cdots<m_{n}$ and, thus, its Noether resolution can also be obtained by means of Theorem 6 . Also when $n=3$, $\mathcal{C}_{2}$ is the curve associated to the arithmetic sequence $m_{1}<m_{3}$. For this reason, the rest of this section only concerns the study of the multigraded Noether resolution of $\mathcal{C}_{r}$ for $r \in\{2, \ldots, n-1\}$ and $n \geq 4$.

Remark 2. Denote by $\mathcal{C}_{n}$ and $\mathcal{C}_{n+1}$ the Zariski closure of $\pi_{n}(\mathcal{C})$ and $\pi_{n+1}(\mathcal{C})$ respectively. Then, both $\mathcal{C}_{n}$ and $\mathcal{C}_{n+1}$ are projective monomial curves associated to arithmetic sequences and, thus, their Noether resolutions can also be obtained by means of Theorem 6 More precisely, the corresponding arithmetic sequences are $m_{1}<\cdots<m_{n-1}$ for $\mathcal{C}_{n}$ and $1<2<\cdots<n-1$ for $\mathcal{C}_{n+1}$, i.e., $\mathcal{C}_{n+1}$ is the rational normal curve of degree $n-1$. 
We denote by $\mathcal{P}_{r}$ the semigroup generated by $\mathcal{A}_{r}$ for $r \in\{2, \ldots, n-1\}$ and $n \geq 4$. Proposition 8 shows how to get the semigroups $\mathcal{P}_{r}$ from $\mathcal{S}$. In the proof of this result we will use the following two lemmas, both of them can be directly deduced from [Bermejo et al. (2017), Lemma 2.1].

Lemma 2. Set $q:=\left\lfloor\left(m_{1}-1\right) /(n-1)\right\rfloor \in \mathbb{N}$; then,

(a) $q+d+1=\min \left\{b \in \mathbb{Z}^{+} \mid b m_{1} \in \sum_{i=2}^{n} \mathbb{N} m_{i}\right\}$

(b) $q+1=\min \left\{b \in \mathbb{Z}^{+} \mid b m_{n} \in \sum_{i=1}^{n-1} \mathbb{N} m_{i}\right\}$

(c) $(q+d) a_{1}+a_{i}=a_{l+i}+q a_{n}+d a_{n+1}$ for all $i \in\{1, \ldots, n-l\}$, where $l:=m_{1}-q(n-1) \in$ $\{1, \ldots, n-1\}$.

Lemma 3. For all $r \in\{2, \ldots, n-1\}$, we have that $m_{r} \in \sum_{i \in\{1, \ldots, n\} \backslash\{r\}} \mathbb{N} m_{i}$ if and only if $r>m_{1}$.

Proposition 8. Set $q:=\left\lfloor\left(m_{1}-1\right) /(n-1)\right\rfloor$ and $l:=m_{1}-q(n-1)$. If $r \leq m_{1}$, then

(a.1) for $r=2$,

$$
\mathcal{S} \backslash \mathcal{P}_{2}= \begin{cases}\left\{\mu a_{1}+a_{2}+\lambda a_{n+1} \mid 0 \leq \mu \leq q+d-1, \lambda \in \mathbb{N}\right\}, & \text { if } l \neq n-1, \\ \left\{\mu a_{1}+a_{2}+\lambda a_{n+1} \mid 0 \leq \mu \leq q+d, \lambda \in \mathbb{N}\right\}, & \text { if } l=n-1,\end{cases}
$$

(a.2) for $r \in\{3, \ldots, n-2\}, \mathcal{S} \backslash \mathcal{P}_{r}=\left\{a_{r}+\lambda a_{n+1} \mid \lambda \in \mathbb{N}\right\}$, and

(a.3) for $r=n-1$,

$$
\mathcal{S} \backslash \mathcal{P}_{n-1}= \begin{cases}\left\{a_{n-1}+\mu a_{n}+\lambda a_{n+1} \mid 0 \leq \mu \leq q-1 \text { or } 0 \leq \lambda \leq d-1\right\}, & \text { if } l \neq n-1, \\ \left\{a_{n-1}+\mu a_{n}+\lambda a_{n+1} \mid 0 \leq \mu \leq q \text { or } 0 \leq \lambda \leq d-1\right\}, & \text { if } l=n-1 .\end{cases}
$$

If $r>m_{1}$, then

(b.1) for $r=2, \mathcal{S} \backslash \mathcal{P}_{2}=\left\{\mu a_{1}+a_{2}+\lambda a_{n+1} \mid 0 \leq \mu, \lambda \leq d-1\right\}$,

(b.2) for $r \in\{3, \ldots, n-2\}, \mathcal{S} \backslash \mathcal{P}_{r}=\left\{a_{r}+\lambda a_{n+1} \mid 0 \leq \lambda \leq d-1\right\}$, and

(b.3) for $r=n-1, \mathcal{S} \backslash \mathcal{P}_{n-1}=\left\{a_{n-1}+\mu a_{n}+\lambda a_{n+1} \mid \mu \in \mathbb{N}, 0 \leq \lambda \leq d-1\right\}$.

Proof. We express every $s \in \mathcal{S}$ as $s=\alpha_{1} a_{1}+\epsilon_{i} a_{i}+\alpha_{n} a_{n}+\alpha_{n+1} a_{n+1}$, with $\alpha_{1}, \alpha_{n}, \alpha_{n+1} \in \mathbb{N}$, $i \in\{2, \ldots, n-1\}$ and $\epsilon_{i} \in\{0,1\}$. Whenever $\epsilon_{i}=0$ or $i \neq r$, it is clear that $s \in \mathcal{P}_{r}$. Hence, we assume that $s=\alpha_{1} a_{1}+a_{r}+\alpha_{n} a_{n}+\alpha_{n+1} a_{n+1}$ and the idea of the proof is to characterize the values of $\alpha_{1}, \alpha_{n}, \alpha_{n+1}$ so that $s \in \mathcal{P}_{r}$ in each case.

Assume first that $r \in\{3, \ldots, n-2\}$ and let us prove (a.2) and (b.2). If $\alpha_{1}>0$ or $\alpha_{n}>0$, the equalities $a_{1}+a_{r}=a_{2}+a_{r-1}$ and $a_{r}+a_{n}=a_{r+1}+a_{n-1}$ yield that $s \in \mathcal{P}_{r}$, so it suffices to consider when $s=a_{r}+\alpha_{n+1} a_{n+1}$. If $r \leq m_{1}$, then by Lemma 3 we get that $s \notin \mathcal{P}_{r}$ because the first coordinate of $s$ is precisely $m_{r}$. This proves (a.2). If $r>m_{1}$ and $\alpha_{n+1} \geq d$, then the equality $a_{r}+d a_{n+1}=d a_{1}+a_{r-m_{1}}$ yields that $s \in \mathcal{P}_{r}$. However, if $\alpha_{n+1}<d$ we are proving that $s \notin \mathcal{P}_{r}$. Suppose by contradiction that $s \in \mathcal{P}_{r}$ and $\alpha_{n+1}<d$, then

$$
s=a_{r}+\alpha_{n+1} a_{n+1}=\sum_{j \in\{1, \ldots, n+1\} \backslash\{r\}} \beta_{j} a_{j}
$$

for some $\beta_{j} \in \mathbb{N}$, then $d \geq 1+\alpha_{n+1}=\sum_{j \in\{1, \ldots, n+1\} \backslash\{r\}} \beta_{j}$. Moreover, observing the first coordinates in (8) we get that $m_{r}=\sum_{j \in\{1, \ldots, n\} \backslash\{r\}} \beta_{j} m_{j}$. Hence, $m_{1}+(r-1) d=\sum_{j\{1, \ldots, n\} \backslash\{r\}} \beta_{j}\left(m_{1}+\right.$ $(j-1) d)$ and, since $\operatorname{gcd}\left\{m_{1}, d\right\}=1$, this implies that $d$ divides $\left(\sum_{j\{1, \ldots, n\} \backslash\{r\}} \beta_{j}\right)-1$, but $0<\left(\sum_{j \in\{1, \ldots, n\} \backslash\{r\}} \beta_{j}\right)-1<d$, a contradiction. Thus $(b .2)$ is proved.

Since the proof of $(a .1)$ is similar to the proof of $(a .3)$ we are not including it here. So let us prove (b.1). Assume that $r=2$. If $\alpha_{n}>0$ the equality $a_{2}+a_{n}=a_{3}+a_{n-1}$ yields that $s \in \mathcal{P}_{2}$, so it suffices to consider when $s=\alpha_{1} a_{1}+a_{r}+\alpha_{n+1} a_{n+1}$. If $\alpha_{1} \geq d$, then the identity $d a_{1}+a_{2}=a_{3}+d a_{n+1}$ yields that $s \in \mathcal{P}_{2}$. For $\alpha_{1}<d$, if $\alpha_{n+1} \geq d$, the equality $\alpha_{1} a_{1}+a_{2}+d a_{n+1}=$ $\left(\alpha_{1}+d+1\right) a_{1}$ also yields that $s \in \mathcal{P}_{2}$. Thus, to conclude (b.l) it only remains to proof that $s \notin \mathcal{P}_{2}$ when $\alpha_{1}, \alpha_{n+1}<d$. Indeed, assume that $\alpha_{1} a_{1}+a_{2}+\alpha_{n+1} a_{n+1}=\sum_{j \in\{1,3, \ldots, n+1\}} \beta_{j} a_{j}$. Observing the first coordinate of the equality we get that $\alpha_{1}+m_{2}=\sum_{j \in\{1,3, \ldots, n\}} \beta_{j} m_{j}$, but $\alpha_{1}+m_{2}<m_{3}<\cdots<m_{n}$, so $\beta_{3}=\cdots=\beta_{n+1}=0$. But this implies that $\beta_{1}=\alpha_{1}+d+1$ and, hence, $\beta_{n+1}<0$, a contradiction. 
Assume now that $r=n-1$. If $\alpha_{1}>0$, the equality $a_{1}+a_{n-1}=a_{2}+a_{n-2}$ yields that $s \in \mathcal{P}_{n-1}$, so it suffices to consider when $s=a_{n-1}+\alpha_{n} a_{n}+\alpha_{n+1} a_{n+1}$. Whenever $s \in \mathcal{P}_{n-1}$, then $s$ can be expressed as $s=\sum_{j \in\{1, \ldots, n-2, n, n+1\}} \beta_{j} a_{j}$, if we consider both expressions of $s$, we get that

(i) $\sum_{j \in\{1, \ldots, n-2, n, n+1\}} \beta_{i}=1+\alpha_{n}+\alpha_{n+1}$, and

(ii) $\sum_{j \in\{1, \ldots, n-2, n\}} \beta_{j} m_{j}=m_{n-1}+\alpha_{n} m_{n}$.

If $\alpha_{n+1}<d$ we are proving that $s \notin \mathcal{P}_{n-1}$. Assume by contradiction that $s \in \mathcal{P}_{n-1}$. From (ii) and Lemma2 2 we deduce that $\beta_{n}<\alpha_{n}$. Moreover, if we expand (ii) considering that $m_{i}=m_{1}+(i-1) d$ for all $i \in\{1, \ldots, n\}$ and that $\operatorname{gcd}\left\{m_{1}, d\right\}=1$, we get that $d$ divides $\sum_{j \in\{1, \ldots, n-2, n\}} \beta_{j}-\alpha_{n}-1=$ $\alpha_{n+1}-\beta_{n+1}$, a contradiction to $0<\alpha_{n+1}-\beta_{n+1}<d$.

Case 1: $m_{1} \geq n-1$. Assume that $s \in \mathcal{P}_{n-1}$. By (ii) and Lemma 3 we have that $\beta_{n}<\alpha_{n}$, so there exists $j_{0} \in\{1, \ldots, n-2\}$ such that $\beta_{j_{0}}>0$. As a consequence, if we add $d-\beta_{n} m_{n}$ in both sides of (ii) we get that $\left(\alpha_{n}+1-\beta_{n}\right) m_{n}=\sum_{j \in\{1 \ldots, n-2\}} \beta_{j} m_{j}-m_{j_{0}}+m_{j_{0}+1} \in \sum_{j \in\{1, \ldots, n-1\}} \mathbb{N} m_{j}$. Hence, by Lemma 2 we have that $\alpha_{n} \geq \alpha_{n}-\beta_{n} \geq q$. If $l<n-1$, for $\alpha_{n} \geq q, \alpha_{n+1} \geq d$ the equality of Lemma $2(q+d) a_{1}+a_{n-l-1}=a_{n-1}+q a_{n}+d a_{n+1}$ shows that $s \in \mathcal{P}_{n-1}$. This proves (a.3) whenever $l \leq n-1$. If $l=n-1$, for $\alpha_{n} \geq q+1, \alpha_{n} \geq d$, again the equality $(q+d+1) a_{1}=(q+1) a_{n}+d a_{n+1}$ shows that $s \in \mathcal{P}_{n-1}$. It only remains to prove that if $\alpha_{n}=q$; then $s \notin \mathcal{P}_{2}$. Assume by contradiction that $a_{n-1}+q a_{n}+\alpha_{n+1} a_{n+1}=\sum_{j \in\{1, \ldots, n-2, n, n+1\}} \beta_{j} a_{j}$. Then, the first coordinates of this equality yield that $m_{n-1}+q m_{n}=\sum_{j \in\{1, \ldots, n-2, n\}} \beta_{j} m_{j}$ and we deduce by Lemma 3 that $\beta_{n}<q$ and, hence, there exists $j_{0} \in\{1, \ldots, n-2\}$ such that $\beta_{j_{0}}>0$. We denote $\beta_{n-1}:=0, \lambda_{j}:=\beta_{j}$ for all $j \in\{1, \ldots, n\} \backslash\left\{j_{0}, j_{0}-1\right\}, \lambda_{j_{0}}=\beta_{j_{0}}-1$, $\lambda_{j_{0}+1}=\beta_{j_{0}+1}+1$, then adding $d$ in both sides of the equality and using Lemma 2 , we get that $(q+1) m_{n}=(q+d+1) m_{1}=\sum_{j \in\{1, \ldots, n\}} \lambda_{j} m_{j} \in \sum_{i=1}^{n} \mathbb{N} m_{i}$. However, $\lambda_{1} \neq q+d+1$, $\lambda_{n} \neq q+1$, so applying iteratively the equalities $a_{i}+a_{j}=a_{i-1}+a_{j+1}$ for all $2 \leq i \leq j \leq n-1$ we express $\sum_{j \in\{1, \ldots, n\}} \lambda_{j} m_{j}$ as $\mu_{1} m_{1}+\epsilon_{k} m_{k}+\mu_{n} m_{n}$ with $\mu_{1}, \mu_{m} \in \mathbb{N}, k \in\{2, \ldots, n-1\}$, $\epsilon_{k} \in\{0,1\}$. It is clear that $\mu_{1} \neq q+d+1$ and that $\mu_{n} \neq q+1$ and one of those is nonzero, so this contradicts the minimality of $q+d+1$ or $q+1$.

To prove (b.3) it only remains to prove that if $\alpha_{n+1} \geq d$, then $s \in \mathcal{P}_{n-1}$, but this easily follows from the relation $a_{n-1}+d a_{n+1}=d a_{1}+a_{n-1-m_{1}}$.

From the previous result and Proposition 5 it is not difficult to obtain the following corollary, which provides the shifts of the first step of a multigraded Noether resolution of $K\left[\mathcal{P}_{r}\right]$ for all $r \in\{2, \ldots, n-1\}$, namely $\left(\mathcal{P}_{r}\right)_{0}:=\left\{s \in \mathcal{P}_{r} \mid s-a_{n}, s-a_{n+1} \notin \mathcal{P}_{r}\right\}$. Indeed, Corollary 4 describes $\left(\mathcal{P}_{r}\right)_{0}$ from the set $\mathcal{S}_{0}$ given by Theorem 6 ,

Corollary 4. We denote $t_{\mu}:=\mu a_{1}+a_{2}$ for all $\mu \in \mathbb{N}$. If $r \leq m_{1}$, then

(a.1) for $r=2$,

$$
\left(\mathcal{P}_{2}\right)_{0}= \begin{cases}\left(\mathcal{S}_{0} \backslash\left\{t_{\mu} \mid 0 \leq \mu \leq q+d-1\right\}\right) \cup\left\{t_{\mu}+a_{n} \mid 0 \leq \mu \leq q+d-1\right\}, & \text { if } l \neq n-1, \\ \left(\mathcal{S}_{0} \backslash\left\{t_{\mu} \mid 0 \leq \mu \leq q+d\right\}\right) \cup\left\{t_{\mu}+a_{n} \mid 0 \leq \mu \leq q+d\right\}, & \text { if } l=n-1,\end{cases}
$$

(a.2) for $r \in\{3, \ldots, n-2\},\left(\mathcal{P}_{r}\right)_{0}=\left(\mathcal{S}_{0} \backslash\left\{a_{r}\right\}\right) \cup\left\{a_{r}+a_{n}\right\}$,

(a.3) for $r=n-1$,

$$
\left(\mathcal{P}_{n-1}\right)_{0}= \begin{cases}\left(\mathcal{S}_{0} \backslash\left\{a_{n-1}\right\}\right) \cup\left\{a_{n-1}+q a_{n}+d a_{n+1}\right\}, & \text { if } l \neq n-1 \\ \left(\mathcal{S}_{0} \backslash\left\{a_{n-1}\right\}\right) \cup\left\{a_{n-1}+(q+1) a_{n}+d a_{n+1}\right\}, & \text { if } l=n-1\end{cases}
$$

If $r>m_{1}$, then

(b.1) for $r=2,\left(\mathcal{P}_{2}\right)_{0}=\left(\mathcal{S}_{0} \backslash\left\{t_{\mu} \mid 0 \leq \mu \leq d-1\right\}\right) \cup\left\{t_{\mu}+a_{n}, t_{\mu}+d a_{n+1} \mid 0 \leq \mu \leq d-1\right\}$,

(b.2) for $r \in\{3, \ldots, n-2\}$, $\left(\mathcal{P}_{r}\right)_{0}=\left(\mathcal{S}_{0} \backslash\left\{a_{r}\right\}\right) \cup\left\{a_{r}+a_{n}, a_{r}+d a_{n+1}\right\}$, and

(b.3) for $r=n-1,\left(\mathcal{P}_{n-1}\right)_{0}=\left(\mathcal{S}_{0} \backslash\left\{a_{n-1}\right\}\right) \cup\left\{a_{n-1}+d a_{n+1}\right\}$.

From Corollary 4 and Proposition 6 , we get the following characterization of the CohenMacaulay property for this family of semigroup rings taking into account that $D$ in Proposition 6 equals $m_{n}$ in these cases. 
Corollary 5. $K\left[\mathcal{P}_{r}\right]$ is Cohen-Macaulay $\Longleftrightarrow r \leq m_{1}$ or $r=n-1$.

Moreover, as a consequence of Theorem 4 and Corollary 4, we get the following result.

Corollary 6. For all $r \in\{2, \ldots, n-2\}$ and $r>m_{1}$, the Macaulayfication of $K\left[\mathcal{P}_{r}\right]$ is $K[\mathcal{S}]$.

In order to get the whole multigraded Noether resolution of $K\left[\mathcal{P}_{r}\right]$ for all $r \in\{2, \ldots, n-2\}$ and $r>m_{1}$, it remains to study its second step. By Theorem 2 , its shifts are given by the set $\left(\mathcal{P}_{r}\right)_{1}:=\left\{s \in \mathcal{P}_{r} \mid s-a_{n}, s-a_{n+1} \in \mathcal{P}_{r}\right.$ and $\left.s-a_{n}-a_{n+1} \notin \mathcal{P}_{r}\right\}$.

\section{Corollary 7.}

$\left(\mathcal{P}_{r}\right)_{1}= \begin{cases}\left\{\mu a_{1}+a_{2}+a_{n}+d a_{n+1} \mid \mu \in\{0, \ldots, d-1\}\right\}, & \text { if } r=2 \text { and } m_{1}=1, \\ \left\{a_{r}+a_{n}+d a_{n+1}\right\}, & \text { if } r \in\{3, \ldots, n-2\} \text { and } m_{1}<r .\end{cases}$

As a consequence of the above results, we are able to provide the multigraded Noether resolution of $K\left[\mathcal{P}_{r}\right]$ for all $r \in\{2, \ldots, n-1\}$.

Theorem 7. Let $q, l \in \mathbb{N}$ be the integers $q:=\left\lceil\left(m_{1}-1\right) /(n-1)\right\rceil$ and $l:=m_{1}-q(n-1)$. If we set $s_{\lambda}:=\left(\left\lceil\frac{\lambda}{n-1}\right\rceil m_{n}-\lambda d, \lambda d\right) \in \mathbb{N}^{2}$ for all $\lambda \in\left\{0, \ldots, m_{n}-1\right\}$, then the multigraded Noether resolution of $K\left[\mathcal{P}_{r}\right]$ is given by the following expressions:

- For $m_{1} \geq 2$, then

$$
0 \rightarrow\left(\oplus_{\lambda=0, \lambda \notin \Lambda_{1}}^{m_{n}-1} A \cdot s_{\lambda}\right) \oplus\left(\oplus_{\lambda \in \Lambda_{1}} A \cdot\left(s_{\lambda}+a_{n}\right)\right) \rightarrow K\left[\mathcal{P}_{2}\right] \rightarrow 0,
$$

where $\Lambda_{1}:=\{\mu(n-1)-1 \mid 1 \leq \mu \leq q+d+\epsilon\}$, and $\epsilon=1$ if $l=n-1$, or $\epsilon=0$ otherwise.

- For $r \in\{3, \ldots, n-2\}$ and $r \leq m_{1}$, then

$$
0 \rightarrow\left(\oplus_{\lambda=0, \lambda \neq n-r}^{m_{n}-1} A \cdot s_{\lambda}\right) \oplus A \cdot\left(a_{r}+a_{n}\right) \rightarrow K\left[\mathcal{P}_{r}\right] \rightarrow 0
$$

- For $r=n-1 \leq m_{1}$, then

$$
0 \rightarrow\left(\oplus_{\lambda=0, \lambda \neq 1}^{m_{n}-1} A \cdot s_{\lambda}\right) \oplus A \cdot\left(a_{n-1}+(q+\epsilon) a_{n}+d a_{n+1}\right) \rightarrow K\left[\mathcal{P}_{n-1}\right] \rightarrow 0,
$$

where $\epsilon=1$ if $l=n-1$, or $\epsilon=0$ otherwise.

- For $m_{1}=1$, then

$$
0 \rightarrow \oplus_{\lambda \in \Lambda_{2}} A \cdot\left(s_{\lambda}+a_{n}+d a_{n+1}\right) \rightarrow \quad \begin{gathered}
\oplus_{\lambda=0, \lambda \notin \Lambda_{2}}^{m_{n}-1} A \cdot s_{\lambda} \\
\oplus \\
\oplus_{\lambda \in \Lambda_{2}} A \cdot\left(s_{\lambda}+a_{n}\right) \\
\oplus_{\lambda \in \Lambda_{2}} A \cdot\left(s_{\lambda}+d a_{n+1}\right)
\end{gathered} \rightarrow K\left[\mathcal{P}_{2}\right] \rightarrow 0,
$$

where $\Lambda_{2}:=\{\mu(n-1)-1 \mid 1 \leq \mu \leq d\}$.

- For $r \in\{3, \ldots, n-2\}$ and $r>m_{1}$, then

$$
0 \rightarrow A \cdot\left(a_{r}+a_{n}+d a_{n+1}\right) \rightarrow \underset{\substack{\left.\oplus_{\lambda=0, \lambda \neq n-r}^{m_{n}-1} A \cdot s_{\lambda}\right) \\ \oplus}}{A \cdot\left(a_{r}+a_{n}\right) \oplus A \cdot\left(a_{r}+d a_{n+1}\right)} \rightarrow K\left[\mathcal{P}_{r}\right] \rightarrow 0 .
$$

- For $r=n-1>m_{1}$, then

$$
0 \rightarrow\left(\oplus_{\lambda=0, \lambda \neq 1}^{m_{n}-1} A \cdot s_{\lambda}\right) \oplus A \cdot\left(a_{n-1}+d a_{n+1}\right) \rightarrow K\left[\mathcal{P}_{n-1}\right] \rightarrow 0 .
$$

It is worth pointing out that from Theorem 7 and Remark 1, one can obtain the Noether resolution of $K\left[\mathcal{P}_{r}\right]$ with respect to the standard grading. In addition, the description of $\left(\mathcal{P}_{r}\right)_{i}$ for all $r \in\{2, \ldots, n-1\}, i \in\{0,1\}$, allows us to use Remark 1 to provide a formula for the Castelnuovo-Mumford regularity of $K\left[\mathcal{P}_{r}\right]$.

Theorem 8. The Castelnuovo-Mumford regularity of $K\left[\mathcal{P}_{r}\right]$ equals: 


$$
\operatorname{reg}\left(K\left[\mathcal{P}_{r}\right]\right)= \begin{cases}\left\lceil\frac{m_{n}-1}{n-1}\right\rceil+1, & \text { if } r \in\{2, n-1\} \text { and } r \leq m_{1}, \\ 2 d, & \text { if } r=2 \text { and } m_{1}=1, \text { and } \\ \left\lceil\frac{m_{n}-1}{n-1}\right\rceil, & \text { if } r \in\{3, \ldots, n-2\}, \text { or } r=n-1 \text { and } m_{1}<r\end{cases}
$$

Let us illustrate the results of this section with an example.

Example 5. Consider the projective monomial curve given parametrically by:

$$
x_{1}=s t^{6}, x_{2}=s^{5} t^{2}, x_{4}=s^{7}, x_{5}=t^{7} .
$$

We observe that the curve corresponds to $\mathcal{C}_{2}$, where $\mathcal{C}$ is the curve associated to the arithmetic sequence $m_{1}<\cdots<m_{n}$ with $m_{1}=1, d=2$ and $n=4$. Hence, by Theorem 7 we get that the multigraded Noether resolution of $K\left[\mathcal{P}_{2}\right]$ is

$$
\begin{aligned}
& A \oplus A \cdot(1,6) \oplus A \cdot(5,2) \oplus \\
0 \rightarrow A \cdot(10,18) \oplus A \cdot(11,24) \rightarrow & A \cdot(2,12) \oplus A \cdot(6,8) \oplus A \cdot(10,4) \oplus \rightarrow K\left[\mathcal{P}_{2}\right] \rightarrow 0 . \\
& A \cdot(3,18) \oplus A \cdot(11,10) \oplus A \cdot(4,24)
\end{aligned} \rightarrow
$$

By Corollary 3 we get that the multigraded Hilbert series of $K\left[\mathcal{P}_{2}\right]$ is

$$
H S_{K\left[\mathcal{P}_{2}\right]}\left(t_{1}, t_{2}\right)=\frac{1+t_{1} t_{2}^{6}+t_{1}^{2} t_{2}^{12}+t_{1}^{4} t_{2}^{24}+t_{1}^{3} t_{2}^{18}+t_{1}^{5} t_{2}^{2}+t_{1}^{6} t_{2}^{8}+t_{1}^{10} t_{2}^{4}-t_{1}^{10} t_{2}^{18}+t_{1}^{11} t_{2}^{10}-t_{1}^{11} t_{2}^{24}}{\left(1-t_{1}^{7}\right)\left(1-t_{2}^{7}\right)} .
$$

Following Remark $\square$ if we consider the standard grading on $R$, we get the following Noether resolution of $K\left[\mathcal{P}_{2}\right]$ :

$$
0 \rightarrow A(-4) \oplus A(-5) \rightarrow \begin{gathered}
A \oplus A(-1)^{2} \oplus A(-2)^{3} \\
A(-3)^{2} \oplus A(-4)
\end{gathered} \rightarrow K\left[\mathcal{P}_{2}\right] \rightarrow 0
$$

and the following expression for the Hilbert series of $K\left[\mathcal{P}_{2}\right]$ :

$$
H S_{K\left[\mathcal{P}_{2}\right]}(t)=\frac{1+2 t+3 t^{2}+2 t^{3}-t^{5}}{(1-t)^{2}}
$$

We also have that $\operatorname{reg}\left(K\left[\mathcal{P}_{2}\right]\right)=4$.

\section{ACKNOWLEDGEMENTS}

The authors want to thank the anonymous referees for their comments and suggestions that we believe have helped to improve this manuscript. In particular, Section 5 was included to answer a question made by the referees.

The first three authors were supported by the Ministerio de Economía y Competitividad, Spain (MTM2013-40775-P and MTM2016-78881-P).

\section{REFERENCES}

[Abbott et al. (2015)] J. Abbott, A. M. Bigatti, G. Lagorio. CoCoA-5: a system for doing Computations in Commutative Algebra. Available at http://cocoa.dima.unige.it

[Bermejo et al. (2017)] I. Bermejo, E. García-Llorente, I. García-Marco, On projective monomial curves associated to generalized arithmetic sequences. J. Symbolic Comput. 81 (2017), 1-19.

[Bermejo \& Gimenez (2000)] I. Bermejo, Ph. Gimenez, On Castelnuovo-Mumford regularity of projective curves. Proc. Amer. Math. Soc. 128 (2000), no. 5, 1293-1299.

[Bermejo \& Gimenez (2001)] I. Bermejo, Ph. Gimenez, Computing the Castelnuovo-Mumford regularity of some subschemes of $\mathbb{P}_{K}^{n}$ using quotients of monomial ideals, Effective methods in algebraic geometry (Bath, 2000). J. Pure Appl. Algebra bf 164 (2001), no. 1-2, 23-33.

[Decker et al. (2015)] W. Decker, G. M. Greuel, G. Pfister and H. Schöenemann, Singular 4-0-2 A computer algebra system for polynomial computations. http://wWw. singular.uni-kl.de (2015).

[Erdös \& Graham (1972)] P. Erdös and R. L. Graham, On a linear diophantine problem of Frobenius, Acta Arithm. 21 (1972), 399-408.

[García-Sánchez \& Rosales (2002)] P. A. García-Sánchez, J. C. Rosales, On Buchsbaum simplicial affine semigroups. Pacific J. Math. 202 (2002), no. 2, 329-393. 
[Gruson et al. (1983)] L. Gruson, R. Lazarsfeld, C. Peskine, On a theorem of Castelnuovo, and the equations defining space curves, Invent. Math. 72(3) (1983), 491-506.

[Goto et al. (1976)] S. Goto, N. Suzuki, K. Watanabe, On affine semigroups. Japan J. Math. 2 (1976), 1-12.

[Grayson \& Stillman (2015)] D. R. Grayson, M. E. Stillman. Macaulay2, a software system for research in algebraic geometry. Available at http://www.math.uiuc.edu/Macaulay2/

[Hoa (1988)] L.T. Hoa, On Segre products of semigroup rings. Nagoya Math. J., Vol.110 (1988), 113-128.

[Hoa (1991)] L.T. Hoa, The Gorenstein property depends upon characteristic for affine semigroup rings. Arch. Math. 56 (1991), 228-235.

[Kawasaki (2000)] T. Kawasaki, On Macaulayfication of Noetherian schemes. Trans. AMS 352(6), 2517-2552.

[L'vovsky (1996)] S. L'vovsky, On inflection points, monomial curves and hypersurfaces containing projective curves, Math. Ann. 306(4)(1996), 719-735.

[Li et. al (2012)] P. Li, D. P. Patil, L. G. Roberts, Bases and ideal generators for projective monomial curves. Comm. Algebra 40 (2012), no. 1, 173-191.

[Molinelli \& Tamone (1995)] S. Molinelli, G. Tamone, On the Hilbert function of certain rings of monomial curves. J. Pure Appl. Algebra 101 (1995), no. 2, 191-206

[Morales (1985)] M. Morales, Fonctions de Hilbert, genre géométrique d'une singularité quasi homogène CohenMacaulay [Hilbert functions, geometric genus of a Cohen-Macaulay quasihomogeneous singularity]. C. R. Acad. Sci. Paris Sér. I Math. 301 (1985), no. 14, 699-702.

[Morales \& Nhan (2003)] M. Morales, L. T. Nhan, On length of generalized fractions $1 /\left(x_{1}^{n}, \ldots, x_{d}^{n}, 1\right)$. Vietnam J. Math. 31 (2003), no. 4, 359-365 .

[Morales (2007)] M. Morales, On the S2-fication of some toric varieties. Comm. Algebra 35 (2007), no. 1, 24092430.

[Morales (2016)] M. Morales, On Hilbert function and Poincaré series of multigraded rings, work in progress

[Ramírez Alfonsín (2005)] J. L. Ramírez Alfonsín, The Diophantine Frobenius Problem, Oxford Lecture Series in Mathematics and its Applications 30, Oxford University Press, 2005.

[Rosales \& García-Sánchez (2009)] J. C. Rosales y P. A. García-Sánchez, Numerical semigroups, Developments in Mathematics, 20, Springer, New York, 2009.

[Selmer (1977)] E. S. Selmer, On the linear diophantine Problem of Frobenius, J. Reine Angewandte Math. 293/294(1) (1977), 1-17.

[Stanley (1978)] R. P. Stanley, Hilbert Functions of Graded Algebras. Adv. Math. 28:1 (1978), 57-83.

[Sturmfels (1996)] B. Sturmfels, Gröbner Bases and Convex Polytopes. University Lecture Series 8, American Mathematical Society, Providence, RI, 1996.

[Trung \& Hoa (1986)] N. V. Trung, L. T. Hoa, Affine semigroups and Cohen-Macaulay rings generated by monomials. Trans. AMS 298(1) (1986), 145-167.

[Villarreal (2015)] R. H. Villarreal, Monomial Algebras, Second Edition, Monographs and Research Notes in Mathematics. Chapman and Hall/CRC, 2015.

Universidad de la laguna. Facultad de Ciencias. Sección de Matemáticas. Avda. AsTrofísico Francisco SÁnchez, S/N. Apartado de Correos 456. 38200-LA Laguna. Tenerife. Spain.

E-mail address: ibermejo@ull.es

Universidad de la laguna. Facultad de Ciencias. Sección de Matemáticas. Avda. AsTrofísico Francisco Sánchez, S/N. Apartado de Correos 456. 38200-La Laguna. Tenerife. Spain.

E-mail address: evgarcia@ull.es

AiX-Marseille Université, CNRS, LIF UMR 7279, MARseille, France.

E-mail address: ignacio.garcia-marcodif.univ-mrs.fr, iggarcia@ull.es

Université de Grenoble I, Institut Fourier, UMr 5582, B.P.74, 38402 Saint-Martin D'Heres Cedex, Grenoble and ESPE de Lyon, Université de Lyon 1, Lyon, France.

E-mail address: morales@ujf-grenoble.fr 